\title{
On the formulation of a BEM in the Bézier-Bernstein space for the solution of Helmholtz equation
}

\author{
A. Romero ${ }^{a, *}$, P. Galvín ${ }^{a}$, J.C. Cámara-Molina ${ }^{a}$, A. Tadeu ${ }^{b, c}$ \\ ${ }^{a}$ Escuela Técnica Superior de Ingeniería, Universidad de Sevilla, Camino de los Descubrimientos, 41092 Sevilla, Spain \\ ${ }^{b}$ ITeCons - Institute for Research and Technological Development in Construction, Energy, Environment and Sustainability, \\ Rua Pedro Hispano s/ n., 3030-289 Coimbra, Portugal \\ ${ }^{c}$ ADAI - LAETA, Department of Civil Engineering, University of Coimbra, Pólo II, Rua Luís Reis Santos, 3030-788 \\ Coimbra, Portugal
}

\begin{abstract}
This paper proposes a novel boundary element approach formulated on the Bézier-Bernstein basis to yield a geometry-independent field approximation. The proposed method is geometrically based on both computer aid design (CAD) and isogeometric analysis (IGA), but field variables are independently approximated from the geometry. This approach allows the appropriate approximation functions for the geometry and variable field to be chosen. We use the Bézier-Bernstein form of a polynomial as an approximation basis to represent both geometry and field variables. The solution of the element interpolation problem in the Bézier-Bernstein space defines generalised Lagrange interpolation functions that are used as element shape functions. The resulting Bernstein-Vandermonde matrix related to the Bézier-Bernstein interpolation problem is inverted using the Newton-Bernstein algorithm. The applicability of the proposed method is demonstrated solving the Helmholtz equation over an unbounded region in a two-and-a-half dimensional (2.5D) domain.

Keywords: subparametric method, Bézier-Bernstein curve, Newton-Bernstein algorithm, computer-aided design, isogeometric analysis
\end{abstract}

\section{Introduction}

We present a geometry-independent field approximation of the boundary element method (BEM). The proposed formulation shares some characteristics with the standard collocation methods and isogeometric analysis (IGA), exploting the advantages of each method. A similar concept was recently introduced by Atroshchenko and Bordas [1. The two strategies are briefly introduced to put this approach into context.

The standard collocation methods are commonly used in engineering to solve boundary value problems [2]. Typically, the objective is to find a function which satisfies a given differential equation with prescribed boundary conditions. The procedure for reducing the continuous problem to its discrete formulation is the

\footnotetext{
* Corresponding author

Email address: aro@us.es (A. Romero)
} 
discretisation of both domain geometry and field variables. The geometry and problem solution are then both approximated as a linear combination of polynomial basis functions defined on a given mesh. The solution accuracy is conditioned by the domain discretisation. Mesh refinement and polynomial based order increase, $h p$-refinement, are strategies used to improve the problem solution. Mesh refinement is a key process that starts with a CAD model of the domain. Each successive mesh is refined in the CAD model. The analysis procedure could fail when the domain geometry needs to be accurately represented. Nowadays, the number of papers dedicated to the development of the BEM and other mesh reduction methods covers a wide field of applications [3 5]. These advances include the development boundary elements for solving thin-walled structures [6] or meshless based methods [7, 8, among others.

In the past decade isogeometric analysis (IGA) has been proposed to deal with geometry approximation. IGA has proved to be very attractive in solving a wide range of problems because it can represent geometries exactly [9 13. IGA was first introduced by Hughes et al. [14] "to be geometrically exact no matter how coarse the discretisation". Also, it simplifies successive mesh refinement since CAD communication is not necessary once a coarse mesh has been defined. It is thus a flexible method that dispenses the polynomial geometry approximation. The major findings reported in [14] are summarised as follows: i) IGA formulation is based on non-uniform rational B-splines basis (NURBS); ii) a mesh for a NURBS patch is defined by the knot vectors and the span between knots divides the representation domain into elements; iii) the geometry is defined as a linear combination of B-spline basis with control points or de Boor points; $i v$ ) field variables are represented by the same function as the geometry by control variables; $v$ ) mesh refinement is based on knot insertion and order elevation techniques without CAD model interaction; vi) element matrices are integrated for each NURBS patch and they are assembled into a global matrix.

One of the main drawbacks of IGA is that the geometry is represented in terms of control points that do not necessarily belong to the boundary, so related control variables do not have a clear physical meaning. Moreover, defining boundary conditions is not straightforward because control variables do not lie on the boundary. The application of inhomogeneous Dirichlet boundary conditions might affect the solution accuracy [14, 15]. Some authors have proposed alternatives based on constraining equations, Lagrange multipliers, penalty methods, or some other method. This fact complicates the isogeometric formulation of the BEM, however, since nodal collocation points do not belong to the boundary [16 19. Alternatively, the Greville abscissae are used to define the collocation points [20].

The proposed strategy can be understood as a formulation that lies between the standard collocation method and the isogeometric analysis. The main thrust of this formulation is the use of an independent basis to represent the geometry and the field variables [1]. This approach was successfully used before the isogeometric development. The works of Zieniuk [21, Zieniuk and Boltuc [22], and Zieniuk and Szerszeń [23], among others, proposed the concept of parametric integral equation system (PIES) for accurate representation of boundary geometry. In these works, the boundary geometry was described as a parametric 
function that was included in the PIES kernels, rather than in the boundary integral as is done in standard BEM. Then, the solution took into account the boundary geometry defined by a parametric function as it is usually done in CAD. Moreover, the authors overcame the difficulties of the original IGA related to the isoparametric concept, computing the solution of PIES by an independent polynomial basis for the field variable approximation 22 .

In this work, we started with the idea that a B-spline can be converted to Bézier curve using the Boehm algorithm 24]: a B-spline of order $n$ is transformed to a composite Bézier curve by inserting knots until all interior knots have a multiplicity equal to $n-1$. Therefore, taking this identity into account, the proposed method will be derived in the Bézier-Bernstein space hereinafter. The geometry is represented by Bézier curves defined as a linear combination of Bernstein basis with control points. The Bernstein basis is widely used in CAD because of its good properties for building and modifying geometric shapes 25. On the other hand, the use of orthogonal basis allows simple and efficient field approximation. To define an orthogonal basis for the field interpolation, therefore, a least-square problem in terms of Bernstein-Vandermonde matrix is generated by the interpolation constraints whose solution is a Lagrange interpolant relative to the Bernstein basis [26, 27]. This methodology is an efficient and robust approach that combines the Bernstein basis for geometry representation and the Lagrange interpolating polynomial relative to the Bernstein basis for the field approximation.

The proposed method is powerful because it includes the main characteristics of standard BEM formulation and isogeometric analysis: $i$ ) the geometry is exactly defined as Bézier curves; $i i)$ field variables are represented at nodal points instead of control variables, which enables easy definition of the boundary conditions; iii) $h$-refinement is performed directly on the geometry without CAD interaction, as it is performed by IGA; and $i v$ ) $p$-refinement only affects the field approximation, rather than the geometry discretisation. Table 1 summarises the characteristics of the BEM approach, isogeometric methods and the proposed formulation of the BEM.

In this paper, the capability of the proposed method is demonstrated for solving the Helmholtz equation in longitudinally invariant problems. This situation is referred to as a two-and-a-half dimensional (2.5D) problem [28, in which the three-dimensional (3D) solution is computed by means of a Fourier transform. The structure of the rest of the paper is as follows. First, the Bézier-Bernstein space is briefly described and the Lagrange interpolant relative to the Bernstein basis is derived. Next, the boundary element formulation in the Bézier-Bernstein space is presented, after which, the proposed methodology is verified by means of two benchmark problems and a numerical example is presented. 
Table 1: Comparison of the boundary element method, based on standard collocation method, isogeometric analysis and geometry-independent field approximation analysis.

\begin{tabular}{l|l|l|l}
\hline & Collocation & Isogeometric & Proposed method \\
\hline Classification & $\begin{array}{l}\text { Sub-/iso-/super- } \\
\text { parametric }\end{array}$ & Isoparametric & $\begin{array}{l}\text { Sub-/iso-/super- } \\
\text { parametric }\end{array}$ \\
\hline \multirow{3}{*}{ Geometry } & Approximate & Exact & Exact \\
& Nodal points & Control points & Control points \\
& Polynomial basis & B-splines & Bézier curves \\
\hline \multirow{2}{*}{ Field } & Nodal variables & Control variables & Nodal variables \\
\hline Boundary condition/ & Interpolation basis & Approximation basis & Interpolation basis \\
Collocation point & Nodes & Control points/ & Nodes \\
\hline
\end{tabular}

\section{Numerical model}

This approach is based on the Helmholtz integral equation for solving 3D interior, exterior and scatterering problems in the frequency-wavenumber domain. The pressure field for the acoustic waveguide at point $\mathbf{x}=\mathbf{x}(x, y, z)$ is computed as the superposition of $2 \mathrm{D}$ solutions with different wavenumbers $\kappa_{z}[29]$ :

$$
p(\mathbf{x}, \omega)=\int_{-\infty}^{+\infty} \widetilde{p}\left(\widetilde{\mathbf{x}}, \kappa_{z}, \omega\right) e^{-\iota \kappa_{z} z} d \kappa_{z}
$$

where $\widetilde{p}\left(\widetilde{\mathbf{x}}, \kappa_{z}, \omega\right)$ is the frequency-wavenumber representation of the sound pressure, $\widetilde{\mathbf{x}}=\mathbf{x}(x, y)$ and $\iota=$ $\sqrt{-1}$.

The boundary integral representation is derived from the Helmholtz equation. The fundamental solution $\widetilde{\Psi}\left(\widetilde{\mathbf{x}}, \kappa_{z}, \omega ; \widetilde{\mathbf{x}}_{i}\right)$ for sound pressure at receiver position $\widetilde{\mathbf{x}}$ due to a source acting at $\widetilde{\mathbf{x}}_{i}$ is the solution to:

$$
\nabla^{2} \widetilde{\Psi}\left(\widetilde{\mathbf{x}}, \kappa_{z}, \omega ; \widetilde{\mathbf{x}}_{i}\right)+\kappa^{2} \widetilde{\Psi}\left(\widetilde{\mathbf{x}}, \kappa_{z}, \omega ; \widetilde{\mathbf{x}}_{i}\right)=-\delta(r)
$$

where $\kappa=\omega / c_{f}$ is the problem wavenumber, $c_{f}$ is the sound propagation velocity, $\delta$ is the Dirac delta function and $r=\left\|\widetilde{\mathbf{x}}_{i}-\widetilde{\mathbf{x}}\right\|$ is the distance from the source to the receiver. The solution to this equation defines the fundamental solution for an unbounded region in the frequency-wavenumber:

$$
\widetilde{\Psi}\left(\widetilde{\mathbf{x}}, \kappa_{z}, \omega ; \widetilde{\mathbf{x}}_{i}\right)=-\frac{\iota}{4} H_{0}^{(2)}\left(\kappa_{\alpha} r\right)
$$

where $\kappa_{\alpha}=\sqrt{\kappa^{2}-\kappa_{z}^{2}}$ and $H_{0}^{(2)}$ is the Hankel function.

The following sections deal with: $i$ ) the Bézier-Bernstein space for geometry representation, ii) the Lagrange interpolant relative to Bernstein basis for variable field approximation, and iii) the boundary element formulation in the Bézier-Bernstein space. 


\subsection{The Bézier-Bernstein space}

The Bernstein polynomial basis was introduced to prove the Weierstrass theorem: given any continuous function $f(x)$ on an interval $[a, b]$ and a tolerance $\epsilon>0$, there is a polynomial $P_{n}(x)$ of sufficiently degree $n$, such that 25]:

$$
\left|f(x)-P_{n}(x)\right| \leq \epsilon, \quad x \in[a, b]
$$

The Bernstein basis of degree $n$ is defined over the interval $t \in[0,1]$ as:

$$
B_{k}^{n}(t)=\left(\begin{array}{l}
n \\
k
\end{array}\right) t^{k}(1-t)^{n-k}, \quad k=0, \ldots, n
$$

The change of variable to map $x \in[a, b]$ to $t \in[0,1]$ allows the definition of the Bernstein polynomial related to a continuous function $f(t)$ as follows:

$$
P_{n}(t)=\sum_{k=0}^{n} f\left(\frac{k}{n}\right) B_{k}^{n}(t)
$$

Then, Equation (6) converges to $f(t)$ as $n$ goes to infinity due to the Bernstein basis being non-negative and forming a partition unity, although the polynomial form does not interpolate the sample values $f(k / n)$. The basis defined by Equation (5) is motivated by its capacity to represent a polynomial in Bernstein form [26] through coefficients $b_{k}$ :

$$
P_{n}(t)=\sum_{k=0}^{n} b_{k} B_{k}^{n}(t)
$$

The Bernstein basis has partition unity property, is defined as non-negative and symmetric about the interval mid-point $\left(B_{n-k}^{n}(1-t)=B_{n}^{k}(t)\right)$. Also, the Bernstein form satisfies the following recursive definition:

$$
B_{k}^{n}(t)=(1-t) B_{k}^{n-1}(t)+t B_{k-1}^{n-1}(t)
$$

The Bernstein basis derivatives are defined by:

$$
\frac{d}{d t} B_{k}^{n}(t)=n\left[B_{k-1}^{n-1}(t)-B_{k}^{n-1}(t)\right]
$$

where $B_{-1}^{n-1}(t)=0$ and $B_{n-1}^{n}(t)=0$. Thus, the derivative of a polynomial in Bernstein form is obtained by combining Equations (7) and (9):

$$
\frac{d}{d t} P_{n}(t)=\sum_{k=0}^{n-1} n\left[b_{k+1}-b_{k}\right] B_{k}^{n-1}(t)
$$


The application of polynomials in Bernstein form growth with the development of Bézier curves $\mathbf{r}_{n}(t)$ in computer-aided design:

$$
\mathbf{r}_{n}(t)=\sum_{k=0}^{n} \mathbf{b}_{k} B_{k}^{n}(t)
$$

where $\mathbf{b}_{k}$ are the control points used to approximate the geometry. A control polygon is obtained by connecting the control points and this is used to modify the curve geometry.

The de Casteljau algorithm is often used for evaluating and splitting a Bézier curve $\mathbf{r}_{n}(t)$ at a given point $t$ [25]. The recursive property of the Bernstein basis defines successive control points $\mathbf{b}_{k}^{l}(t)$ as a recursive linear interpolation:

$$
\mathbf{b}_{k}^{l}(t)=(1-t) \mathbf{b}_{k}(t)^{l-1}+t \mathbf{b}_{k+1}(t)^{l-1}, \quad l=1, \ldots, n, \quad k=0,1, \ldots, n-l
$$

Starting with the control polygon given by control points $\mathbf{b}_{k}^{0}=\mathbf{b}_{k}$, de Casteljau algorithm defines a new polygon whose vertex is smaller than the original one. The last point in the recursive procedure corresponds to the Bézier representation $\mathbf{r}(t)=\mathbf{b}_{n}^{0}(t)$. Moreover, two new Bézier curves can be defined by control points $\left\{\mathbf{b}_{k}^{l}(t)\right\}_{k=0}^{l=0, \ldots, n}$ and $\left\{\mathbf{b}_{k}^{l}(t)\right\}_{k=n-l}^{l=n, \ldots, 0}$.

Although the de Casteljau algorithm allows an easy evaluation of a Bézier curve, it is computationally expensive. An efficient curve computation is achieved using the polar form (or blossom) of a Bézier curve $\mathbf{r}_{n}(t)$ [30, which defines a multi-affine transformation satisfying:

$$
\mathbf{b}_{k}=\mathbf{R}(\underbrace{0, \ldots, 0}_{n-k}, \underbrace{1, \ldots, 1}_{k})
$$

where $\mathbf{R}\left(t_{1}, \ldots, t_{n}\right)$ is computed as:

$$
\mathbf{R}\left(t_{1}, \ldots, t_{n}\right)=\sum_{\substack{I \cap J=\emptyset \\ I \cup J=\{1,2, \ldots, n\}}} \prod_{i \in I}\left(1-t_{i}\right) \prod_{j \in J} t_{j} \mathbf{b}_{|J|}
$$

Thus, a polynomial in Bernstein form can be formulated in polar form substituting Equation (13) into Equation 11 as follows:

$$
\mathbf{r}_{n}(t)=\sum_{k=0}^{n} \mathbf{R}(\underbrace{0, \ldots, 0}_{n-k}, \underbrace{1, \ldots, 1}_{k}) B_{k}^{n}(t)=\mathbf{R}(t, \ldots, t)
$$

The polar form of a Bézier curve $\mathbf{r}_{n}(t)$ of degree $n$ defines a multi-affine polynomial $\mathbf{R}\left(t_{1}, \ldots, t_{n}\right)$ with $n$ variables that satisfy $\mathbf{r}_{n}(t)=\mathbf{R}(t, \ldots, t)$. Moreover, the following expression can be derived from Equations 
(12) and (13):

$$
\begin{aligned}
\mathbf{R}(\underbrace{0, \ldots, 0}_{n-(l+k)}, \underbrace{t, \ldots, t}_{l}, \underbrace{1, \ldots, 1}_{k}) & =(1-t) \mathbf{R}(\underbrace{0, \ldots, 0}_{n-(l+k)+1}, \underbrace{t, \ldots, t}_{l-1}, \underbrace{1, \ldots, 1}_{k}) \\
& +t \mathbf{R}(\underbrace{0, \ldots,}_{n-(l+k)}, \underbrace{t, \ldots, t}_{l-1}, \underbrace{1, \ldots, 1}_{k+1}), \quad l=1, \ldots, n, \quad k=0,1, \ldots, n-l
\end{aligned}
$$

which it is in agreement with Equation 14 when $l=n$.

The new Bézier curves defined by control points $\left\{\mathbf{b}_{k}^{l}\left(t^{*}\right)\right\}_{k=0}^{l=0, \ldots, n}$ and $\left\{\mathbf{b}_{k}^{l}\left(t^{*}\right)\right\}_{k=n-l}^{l=n, \ldots, 0}$ at a given point $t^{*}$ according to the de Casteljau algorithm are represented by:

$$
\mathbf{r}_{n}^{*}(t)=\sum_{k=0}^{n} \mathbf{R}(\underbrace{0, \ldots, 0}_{n-k}, \underbrace{t^{*}, \ldots, t^{*}}_{k}) B_{k}^{n}(t)
$$

and,

$$
\mathbf{r}_{n}^{*}(t)=\sum_{k=0}^{n} \mathbf{R}(\underbrace{t^{*}, \ldots, t^{*}}_{n-k}, \underbrace{1, \ldots, 1}_{k}) B_{k}^{n}(t)
$$

respectively.

The BEM formulated within the Bézier-Bernstein space needs the derivative of $\mathbf{r}_{n}(t)$ to compute tangent and normal vectors and the Jacobian of the transformation. The first derivative is evaluated according to Equations (10) and (11) as:

$$
\frac{d}{d t} \mathbf{r}_{n}(t)=\sum_{k=0}^{n-1} n\left[\mathbf{b}_{k+1}-\mathbf{b}_{k}\right] B_{k}^{n-1}(t)
$$

This expression can be rewritten using the polar form as follows [31:

$$
\frac{d}{d t} \mathbf{r}_{n}(t)=\frac{d}{d t} \mathbf{R}(t, \ldots, t)=n(\mathbf{R}(\underbrace{t, \ldots, t}_{n-1}, 1)-\mathbf{R}(0, \underbrace{t, \ldots, t}_{n-1}))
$$

Once the first derivative of $\mathbf{r}_{n}(t)$ is computed, the tangent and normal vectors and the Jacobian are easily obtained.

Cubic Bézier approximations are widely used to represent smooth patches in CAD. More complex shapes would be defined by either increasing the curve order or using a piecewise Bézier curve. In this case, control points must satisfy $C^{1}$ and $C^{2}$ end-point interpolation conditions [32, 33. Figure 1 shows a third-order 
Bézier patch and its related control points represented in polar form as:

$$
\begin{aligned}
\mathbf{R}\left(t_{1}, t_{2}, t_{3}\right) & =\left[\left(1-t_{1}\right)\left(1-t_{2}\right)\left(1-t_{3}\right)\right] \mathbf{b}_{0} \\
& +\left[\left(1-t_{2}\right)\left(1-t_{3}\right) t_{1}+\left(1-t_{1}\right)\left(1-t_{3}\right) t_{2}+\left(1-t_{1}\right)\left(1-t_{2}\right) t_{3}\right] \mathbf{b}_{1} \\
& +\left[\left(1-t_{3}\right) t_{1} t_{2}+\left(1-t_{1}\right) t_{2} t_{3}+\left(1-t_{2}\right) t_{3} t_{1}\right] \mathbf{b}_{2} \\
& +\left[t_{1} t_{2} t_{3}\right] \mathbf{b}_{3}
\end{aligned}
$$

The resulting new curves are defined through control points $\{\mathbf{R}(0,0,0), \mathbf{R}(0,0, t), \mathbf{R}(0, t, t), \mathbf{R}(t, t, t)\}$ and $\{\mathbf{R}(t, t, t), \mathbf{R}(t, t, 1), \mathbf{R}(t, 1,1), \mathbf{R}(1,1,1)\}$ according to the de Casteljau algorithm.

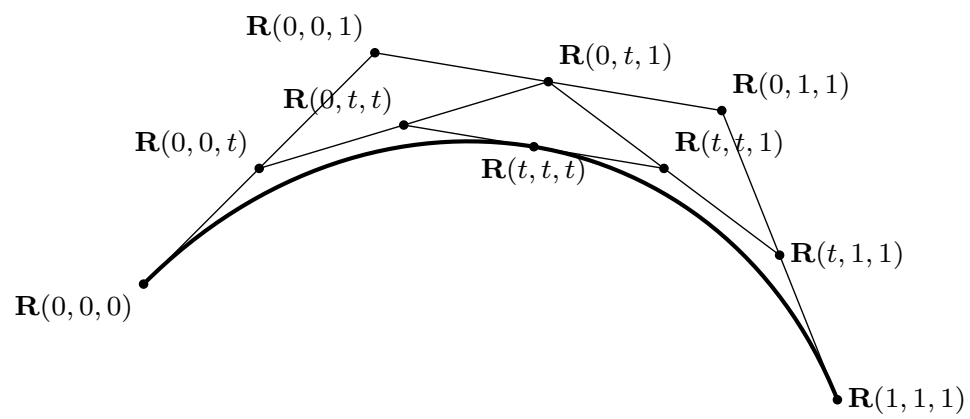

Figure 1: Third-order Bézier patch.

The geometry approximation presented in this section is used to formulate the BEM in the BézierBernstein space. The boundary geometry is defined by patches with an arbitrary approximation degree, that depends on the geometry shape complexity. Then, each patch is subdivided into elements according to $h$-refinement.

Section 3.2 analyses the accuracy of the proposed method studying the scattered wavefield by a circular inclusion using $C^{1}$ third and $C^{2}$ fourth order Bézier curves.

\subsection{Field approximation}

The Bézier-Bernstein space allows the approximation of a curve geometry through control points. Moreover, the element shape function can be derived from the Lagrange interpolant relative to the Bernstein basis presented in the previous section. The field approximation consists of variable interpolation from known nodal values through element shape functions. The approximation of a field variable is given by finding a polynomial $P_{n}(x)$ that interpolates a function $f(x)$ at discrete points $x_{j}$ :

$$
P_{n}\left(x_{i}\right)=f\left(x_{i}\right), \quad i=0, \ldots, n
$$

where $x_{i}$ are $n+1$ nodes and $f\left(x_{i}\right)$ are given data. The problem has a solution that only depends on the interpolation data. The solution can be defined in Lagrange form [34], but each evaluation of $P_{n}(x)$ 
results in an $\mathcal{O}\left(n^{2}\right)$ complexity algorithm and its computation is numerically unstable [35. Berrut and Trefethen [34] presented a barycentric Lagrange formula which has $\mathcal{O}(n)$ complexity. Moreover, they stated that setting equidistant nodes for high-order interpolation polynomials causes the appearence of the socalled Runge phenomenon, which results in a highly ill-conditioned basis. The polynomial basis becomes a well-conditioned process for polynomial interpolation when elements with nodes mainly concentrated at the endpoints with an asymptotic density proportional to $(1-x)^{-1 / 2}$ are used. Legendre-Gauss-Lobatto (LGL) integration points are used in spectral formulations for this purpose [36]. Alternatively, we propose simpler node distributions such as the families of Chebyshev points to develop the BEM in the Bézier-Bernstein space.

Chebyshev points of the first kind are given by:

$$
x_{i}=\cos \left(\frac{(2 i+1) \pi}{2 n+2}\right), \quad i=0, \ldots, n
$$

and, of the second kind by:

$$
x_{i}=\cos \left(\frac{i \pi}{n}\right), \quad i=0, \ldots, n
$$

In these cases, if $f(x)$ is a smooth function defined at the interval $[0,1]$ interpolated by polynomials in Chebyshev points, the rate of convergence is remarkably faster. The interpolant $P_{n}$ satisfies an error estimation, thus:

$$
\max _{x \in[0,1]}\left|f(x)-P_{n}(x)\right| \leq C K^{-n}
$$

for constants $C$ and $K>1$ [37.

The Lagrange interpolant relative to the Bernstein basis has become useful for high-order element development [38]. Although a polynomial in Bernstein form satisfies $P_{n}(0)=f(0)$ and $P_{n}(1)=f(1)$, these properties do not remain valid at internal element points as has been stated. Therefore, it is necessary to properly modify the control points $b_{k}$ to ensure the following condition:

$$
P_{n}\left(x_{i}\right)=\sum_{k=0}^{n} b_{k} B_{k}^{n}\left(x_{i}\right)=f\left(x_{i}\right)
$$

This condition is commonly expressed as a linear system of equations through the Bernstein-Vandermonde matrix $A_{i j}=B_{i}^{j}\left(x_{i}\right)$, for $i, j=0, \ldots, n$. The control points $\mathbf{b}=\left[b_{0}, \ldots, b_{n}\right]^{T}$ are given by the solution of $\mathbf{A} \mathbf{b}=\mathbf{f}$, where the vector $\mathbf{f}=\left[f\left(x_{0}\right), \ldots, f\left(x_{n}\right)\right]^{T}$ collects the interpolation data.

Some algorithms have been presented for solving Bernstein-Vandermonde linear systems [35, 39 41. Marco and Martínez [39] studied the condition number of linear systems defined by the Bernstein-Vandermonde matrix and concluded that they are exponentially ill-conditioned. In this work, we have used the NewtonBernstein algorithm proposed by Ainsworth and Sánchez [35] to compute the control points. 
The Newton-Bernstein algorithm [35] starts with the Newton form of the interpolant $P_{n}$ at nodes $x_{i}$ :

$$
P_{k}(x)=\sum_{i=0}^{k} f\left[x_{0}, \ldots, x_{k}\right] w_{i}(x), \quad k=0, \ldots, n
$$

where $f\left[x_{0}, \ldots, x_{k}\right]$ is the divided difference defined by the following recursive formula:

$$
f\left[x_{j}, \ldots, x_{k}\right]=\frac{f\left[x_{j+1}, \ldots, x_{k}\right]-f\left[x_{j}, \ldots, x_{k-1}\right]}{x_{k}-x_{j}}, \quad k=j+1, \ldots, n ; \quad j=0, \ldots, n
$$

Thus, the following expressions for control points $w_{k}$ and $b_{k}$, for $k=0, \ldots, n$ are defined:

$$
\begin{aligned}
& w_{k}\left(x_{j}\right)=\frac{j}{k} w_{k-1}\left(x_{j-1}\right)\left(1-x_{k-1}\right)-\frac{k-j}{k} w_{k-1}\left(x_{j}\right) x_{k-1} \\
& b_{k}\left(x_{j}\right)=\frac{j}{k} b_{k-1}\left(x_{j-1}\right)+\frac{k-j}{k} b_{k-1}\left(x_{j}\right)+w_{k}\left(x_{j}\right) f\left[x_{0}, \ldots, x_{k}\right]
\end{aligned}
$$

and for $j=0, \ldots, k$ the latter expressions are $w_{0}\left(x_{0}\right)=1, b_{0}\left(x_{0}\right)=f\left[x_{0}\right], w_{-1}\left(x_{k-1}\right)=b_{-1}\left(x_{k-1}\right)=0$ and $w_{k}\left(x_{k-1}\right)=b_{k}\left(x_{k-1}\right)=0$.

The Lagrange polynomial $P_{n}$ of order $n$ defined through the Bernstein basis gives $n+1$ interpolation functions $\phi^{j}$ computed after imposing the constrains $P_{n}^{j}\left(x_{k}\right)=\phi^{j}\left(x_{k}\right)=\delta\left(x_{k}-x_{j}\right)$, for $j, k=0, \ldots, n$. Then, $\mathbf{b}$ becomes a matrix of unknown control points and $\mathbf{f}=\mathbf{I}$ is the identity matrix. As an example, control points of the Lagrangian interpolant of degree $n=6$ defined at Chebyshev points of the first kind are:

$$
\mathbf{b}=\left[\begin{array}{rrrrrrr}
1.2679 & -2.5844 & 3.1817 & -2.4066 & 1.0729 & -0.2441 & 0.0161 \\
-0.4083 & 5.6362 & -8.8317 & 7.2028 & -3.2988 & 0.7571 & -0.0500 \\
0.2274 & -3.3523 & 11.7759 & -11.7676 & 5.7761 & -1.3555 & 0.0898 \\
-0.1429 & 2.1429 & -8.6762 & 14.9429 & -8.6762 & 2.1429 & -0.1429 \\
0.0898 & -1.3555 & 5.7761 & -11.7676 & 11.7759 & -3.3523 & 0.2274 \\
-0.0500 & 0.7571 & -3.2988 & 7.2028 & -8.8317 & 5.6362 & -0.4083 \\
0.0161 & -0.2441 & 1.0729 & -2.4066 & 3.1817 & -2.5844 & 1.2679
\end{array}\right]
$$

and those defined at Chebyshev points of the second kind are:

$$
\mathbf{b}=\left[\begin{array}{rrrrrrr}
1.0000 & -3.0556 & 4.4000 & -3.3000 & 1.2222 & -0.1667 & 0.0000 \\
0.0000 & 4.9761 & -8.7681 & 6.9333 & -2.6097 & 0.3573 & 0.0000 \\
0.0000 & -1.3333 & 8.1778 & -8.0000 & 3.2000 & -0.4444 & 0.0000 \\
0.0000 & 0.6667 & -4.6222 & 9.7333 & -4.6222 & 0.6667 & 0.0000 \\
0.0000 & -0.4444 & 3.2000 & -8.0000 & 8.1778 & -1.3333 & 0.0000 \\
0.0000 & 0.3573 & -2.6097 & 6.9333 & -8.7681 & 4.9761 & 0.0000 \\
0.0000 & -0.1667 & 1.2222 & -3.3000 & 4.4000 & -3.0556 & 1.0000
\end{array}\right]
$$

The related interpolation functions are computed for a given point $x$ as $\left[\phi^{1}(x), \ldots, \phi^{j}(x), \ldots, \phi^{n+1}(x)\right]^{T}=$ bA $(x)$. The evaluation of the shape functions also benefits from the computational advantages of using the polar form given in the previous section.

Figure 2 shows the Lagrangian interpolant relative to the Bernstein basis defined at the Chebyshev points defined by control points (31) and (32), besides of those defined at equidistant nodes and at LGL integration 
points. Figure 2. (a) shows the instabilities due to the Runge phenomenon as described in [34], whereas the other interpolation functions behave adequately and can be used as shape functions in the development of the proposed method.

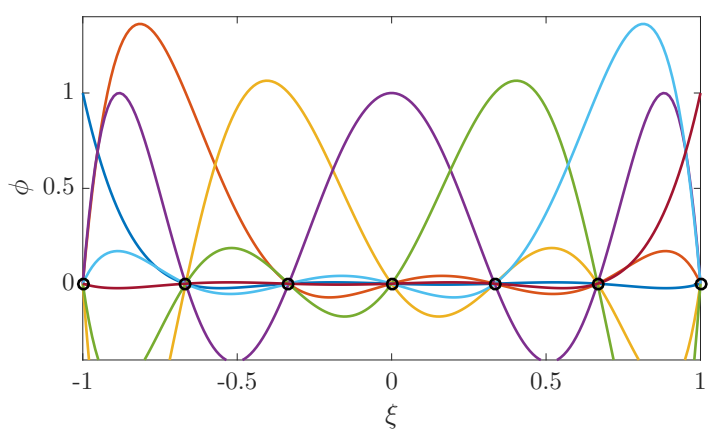

(a)

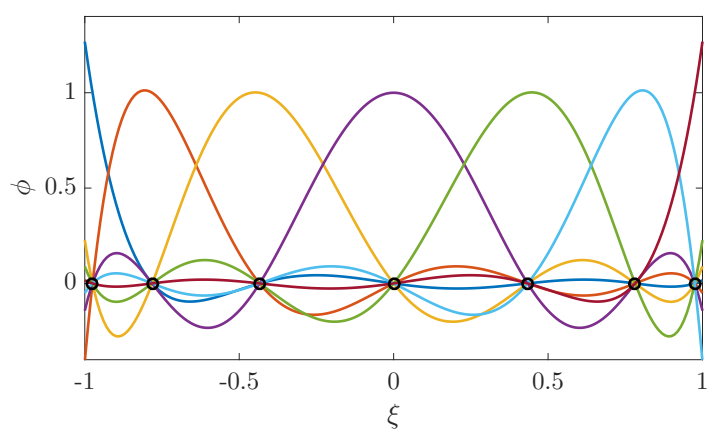

(c)
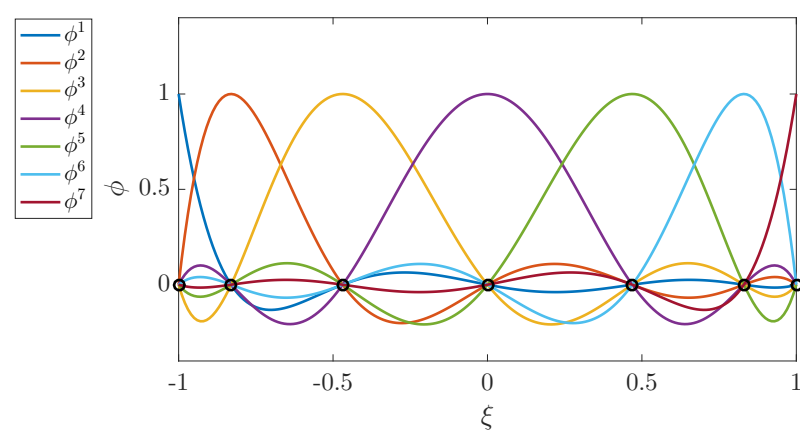

(b)
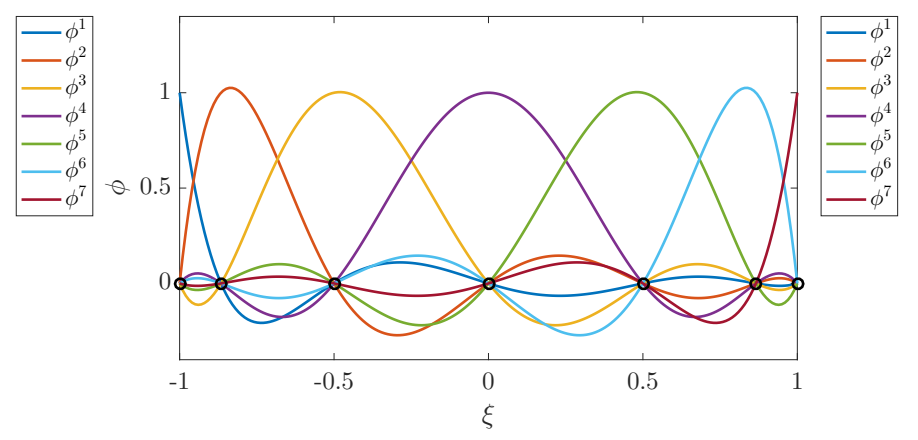

(d)

Figure 2: Element shape functions of order $n=6$ defined at: (a) equidistant nodes, (b) LGL integration points, (c) Chebyshev points of first kind, and (d) Chebyshev points of the second kind. Nodal coordinates are marked by circles.

In this work, we have used two kinds of boundary elements: $i$ ) continuous elements based on a Lagrangian interpolant defined at equidistant nodes, LGL integration points and Chebyshev points of the second kind; and $i$ ) discontinuous elements defined at Chebyshev points of the first kind.

\subsection{Boundary element formulation}

Since the Bézier-Bernstein basis allowed the independent geometry and field variable approximation, the formulation of the BEM in Bézier-Bernstein space is quite straightforward, as in a classical formulation. This section describes the boundary element formulation for potential problems in acoustics [42. The integral representation of the sound pressure in the frequency-wavenumber domain for a point $\widetilde{\mathbf{x}}_{i}$ located at the arbitrary boundary $\Gamma$ can be written as:

$$
c_{i}\left(\widetilde{\mathbf{x}}_{i}\right) \widetilde{p}_{i}\left(\widetilde{\mathbf{x}}_{i}, \kappa_{z}, \omega\right)=-\int_{\Sigma}\left(\iota \rho \omega \widetilde{v}_{i}\left(\widetilde{\mathbf{x}}_{i}, \kappa_{z}, \omega\right) \widetilde{\Psi}\left(\widetilde{\mathbf{x}}, \kappa_{z}, \omega ; \widetilde{\mathbf{x}}_{i}\right)+\widetilde{p}_{i}\left(\widetilde{\mathbf{x}}_{i}, \kappa_{z}, \omega\right) \frac{\partial \widetilde{\Psi}\left(\widetilde{\mathbf{x}}, \kappa_{z}, \omega ; \widetilde{\mathbf{x}}_{i}\right)}{\partial \mathbf{n}}\right) d \Sigma
$$


where $\widetilde{p}\left(\widetilde{\mathbf{x}}_{i}, \kappa_{z}, \omega\right)$ and $\widetilde{v}\left(\widetilde{\mathbf{x}}_{i}, \kappa_{z}, \omega\right)$ are the sound pressure and the particle normal velocity at the cross section $\Sigma$ of the boundary $\Gamma$, respectively. $\widetilde{\Psi}\left(\widetilde{\mathbf{x}}, \kappa_{z}, \omega ; \widetilde{\mathbf{x}}_{i}\right)$ is the solution to the Helmholtz equation at point $\widetilde{\mathbf{x}}$ due to a point source located at $\widetilde{\mathbf{x}}_{i}$ (Equation (3)). The integral-free term $c_{i}\left(\widetilde{\mathbf{x}}_{i}\right)$ depends only on the boundary geometry at the collocation point $\widetilde{\mathbf{x}}_{i}$.

The boundary is discretised into $N$ elements with $\Sigma=\bigcup_{j=1}^{N} \Sigma^{j}$, leading to a boundary approximation of the element field variables using the interpolation shape function $\phi$ of order $p$ :

$$
\begin{aligned}
& \sum_{i=1}^{p+1} \phi^{i} \widetilde{p}^{i}=\phi \widetilde{\mathbf{p}}^{e} \\
& \sum_{i=1}^{p+1} \phi^{i} \widetilde{v}^{i}=\phi \widetilde{\mathbf{v}}^{e}
\end{aligned}
$$

Hereinafter, we denote the element order by $p$ to distinguish from the Bézier curve degree $n$. We shall abuse notation and use $p$ for the element order as well as the sound pressure in Equation (1). It will be clear from the context which one $p$ refers to.

The proposed method employs the Lagrange interpolants relative to the Bernstein basis described in Section 2.2 as approximation shape functions. Thus, Equation $\sqrt{33}$ is written as:

$$
c_{i} \widetilde{p}_{i}=-\sum_{j=1}^{Q}\left[\iota \rho \omega\left\{\int_{\Sigma^{j}} \phi^{j} \widetilde{\Psi} d \Sigma\right\} \widetilde{v}^{j}+\left\{\int_{\Sigma^{j}} \phi^{j} \frac{\partial \widetilde{\Psi}}{\partial \mathbf{n}} d \Sigma\right\} \widetilde{p}^{j}\right]
$$

where $Q$ is the total number of nodes at the boundary and $\Sigma^{j}(\widetilde{\mathbf{x}})$ stands for the elements which contain the node $j$. The following integrals are defined for each collocation point $\widetilde{\mathbf{x}}_{i}$ and integration element $\Sigma^{j}$ :

$$
\begin{aligned}
& \mathbf{h}_{i}^{j}\left(\widetilde{\mathbf{x}}, \kappa_{z}, \omega ; \widetilde{\mathbf{x}}_{i}\right)=\int_{\Sigma^{j}} \phi^{j} \frac{\partial \widetilde{\Psi}}{\partial \mathbf{n}} d \Sigma \\
& \mathbf{g}_{i}^{j}\left(\widetilde{\mathbf{x}}, \kappa_{z}, \omega ; \widetilde{\mathbf{x}}_{i}\right)=\int_{\Sigma^{j}} \phi^{j} \widetilde{\Psi} d \Sigma
\end{aligned}
$$

Equations (37) and (38) define the element matrices as in standard BEM formulation. Now, the BézierBernstein space is used to describe the exact element geometry as $\Sigma^{j}(\widetilde{\mathbf{x}})=\mathbf{r}_{n}^{j}(t)$. Hence, the later integrals along a boundary element $\Sigma^{j}$ are rewritten in the univariate basis $t \in[0,1]$ as:

$$
\int_{\Sigma^{j}} \widetilde{f}\left(\widetilde{\mathbf{x}}, \kappa_{z}, \omega ; \widetilde{\mathbf{x}}_{i}\right) d \Sigma=\int_{0}^{1} f\left(\widetilde{\mathbf{x}}(t), \kappa_{z}, \omega ; \widetilde{\mathbf{x}}_{i}\right)\left|\frac{d}{d t} \mathbf{r}_{n}^{j}(t)\right| d t
$$

where $\widetilde{f}\left(\widetilde{\mathbf{x}}, \kappa_{z}, \omega ; \widetilde{\mathbf{x}}_{i}\right)$ represents the kernels in Equations 37) and 387. Thus, Equation 39 is transformed into a symmetric integration interval $\xi \in[-1,1]$ to employ a Gauss-Legendre quadrature as:

$$
\int_{\Sigma^{j}} \tilde{f}\left(\widetilde{\mathbf{x}}, \kappa_{z}, \omega ; \widetilde{\mathbf{x}}_{i}\right) d \Sigma=\int_{-1}^{1} \widetilde{f}\left(\widetilde{\mathbf{x}}(\xi), \kappa_{z}, \omega ; \widetilde{\mathbf{x}}_{i}\right)\left|\frac{d}{d t} \mathbf{r}_{n}^{j}(t)\right| \frac{d t(\xi)}{d \xi} d \xi
$$

The relation $2 t=\xi+1$ defines the Jacobian of the transformation $d t / d \xi=1 / 2$. 
Finally, Equations (37) and (38) are expressed as follows:

$$
\begin{aligned}
\mathbf{h}_{i}^{j}\left(\widetilde{\mathbf{x}}, \kappa_{z}, \omega ; \widetilde{\mathbf{x}}_{i}\right) & =\frac{1}{2} \int_{-1}^{1} \phi^{j} \frac{\partial \widetilde{\Psi}}{\partial \mathbf{n}}\left|\frac{d}{d t} \mathbf{r}_{n}^{j}(t)\right| d \xi \\
\mathbf{g}_{i}^{j}\left(\widetilde{\mathbf{x}}, \kappa_{z}, \omega ; \widetilde{\mathbf{x}}_{i}\right) & =\frac{1}{2} \int_{-1}^{1} \phi^{j} \widetilde{\Psi}\left|\frac{d}{d t} \mathbf{r}_{n}^{j}(t)\right| d \xi
\end{aligned}
$$

The system of equations for all the boundary elements becomes:

$$
\widetilde{\mathbf{H}}\left(\widetilde{\mathbf{x}}, \kappa_{z}, \omega ; \widetilde{\mathbf{x}}_{i}\right) \widetilde{p}\left(\widetilde{\mathbf{x}}, \kappa_{z}, \omega\right)=\widetilde{\mathbf{G}}\left(\widetilde{\mathbf{x}}, \kappa_{z}, \omega ; \widetilde{\mathbf{x}} i\right) \widetilde{v}\left(\widetilde{\mathbf{x}}, \kappa_{z}, \omega\right)
$$

where $\widetilde{\mathbf{H}}\left(\widetilde{\mathbf{x}}, \kappa_{z}, \omega ; \widetilde{\mathbf{x}}_{i}\right)$ and $\widetilde{\mathbf{G}}\left(\widetilde{\mathbf{x}}, \kappa_{z}, \omega ; \widetilde{\mathbf{x}}_{i}\right)$ are the fully non-symmetrical boundary element system matrices, and $\widetilde{p}\left(\widetilde{\mathbf{x}}, \kappa_{z}, \omega\right)$ and $\widetilde{v}\left(\widetilde{\mathbf{x}}, \kappa_{z}, \omega\right)$ are the sound pressure and particle normal velocity at the boundary $\Sigma$, respectively.

The boundary integrals are computed using a standard Gauss-Legendre quadrature with $4(p+1)$ integration points whenever the collocation point is sufficiently distant from the integration element. Otherwise, the solution of singular or weakly singular integrals is numerically computed using a smoothing transformation by means of a Gauss-Legendre quadrature [43. Figure 3 shows a scheme for the treatment of singular and weakly singular integrals. This figure represents a collocation point $\widetilde{\mathbf{x}}_{i}$ and an integration element $\Sigma^{j}$. The projection point $t\left(\widetilde{\mathbf{x}}_{i}\right)$ is found from $\widetilde{\mathbf{x}}_{i}$ as the point that minimizes the distance $r\left(\widetilde{\mathbf{x}}_{i}\right)$ to the integration element. Obviously, if the collocation point belongs to the integration element, then $t\left(\widetilde{\mathbf{x}}_{i}\right)$ coincides with the coordinate of this node. Once the point $t\left(\widetilde{\mathbf{x}}_{i}\right)$ has been identified, the element integration defined by Equation (39) becomes:

$$
\int_{\Sigma^{j}} \widetilde{f}\left(\widetilde{\mathbf{x}}, \kappa_{z}, \omega ; \widetilde{\mathbf{x}}_{i}\right) d \Sigma=\int_{0}^{t\left(\widetilde{\mathbf{x}}_{i}\right)} f\left(\widetilde{\mathbf{x}}(t), \kappa_{z}, \omega ; \widetilde{\mathbf{x}}_{i}\right)\left|\frac{d}{d t} \mathbf{r}_{n}^{j}(t)\right| d t+\int_{t\left(\widetilde{\mathbf{x}}_{i}\right)}^{1} f\left(\widetilde{\mathbf{x}}(t), \kappa_{z}, \omega ; \widetilde{\mathbf{x}}_{i}\right)\left|\frac{d}{d t} \mathbf{r}_{n}^{j}(t)\right| d t
$$

where the integration path is subdivided into two intervals according to $t\left(\widetilde{\mathbf{x}}_{i}\right)$. These integrals are numerically solved by a smoothing transformation of the form $\widetilde{\mathbf{x}}=\varphi_{l, r}(s)[43$ :

$$
\varphi_{l, r}(s)=\frac{(\alpha+\beta-1) !}{(\alpha-1) !(\beta-1) !} \int_{0}^{s} u^{\alpha-1}(1-u)^{\beta-1} d u, \quad s \in[0,1]
$$

where,

$$
\Xi_{\alpha, \beta}(s)=\int_{0}^{s} u^{\alpha-1}(1-u)^{\beta-1} d u
$$

is computed using the recurrence expression:

$$
\Xi_{\alpha, \beta}(s)=\frac{1}{\alpha+\beta-1}\left[-s^{\alpha-1}(1-s)^{\beta}+(\alpha-1) \Xi_{\alpha-1, \beta}(s)\right], \quad \alpha \geq 2, \beta \geq 1
$$

with the initial value $\Xi_{1,1}(s)=s$. Accurate results for singular integrals are given in the next section, setting $\alpha=3$ and $\beta=1$, and using a number of integration points $10(p+1)$. 
Therefore, Equation (44) becomes:

$$
\begin{aligned}
\int_{\Sigma^{j}} \widetilde{f}\left(\widetilde{\mathbf{x}}, \kappa_{z}, \omega ; \widetilde{\mathbf{x}}_{i}\right) d \Sigma= & -\int_{0}^{1} f\left(\widetilde{\mathbf{x}}\left(\varphi_{l}(s)\right), \kappa_{z}, \omega ; \widetilde{\mathbf{x}}_{i}\right)\left|\frac{d}{d t} \mathbf{r}_{n}^{j}(t)\right| \frac{d t}{d \varphi_{l}} \frac{d}{d s} \varphi_{l}(s) d s \\
& +\int_{0}^{1} f\left(\widetilde{\mathbf{x}}\left(\varphi_{r}(s)\right), \kappa_{z}, \omega ; \widetilde{\mathbf{x}}_{i}\right)\left|\frac{d}{d t} \mathbf{r}_{n}^{j}(t)\right| \frac{d t}{d \varphi_{r}} \frac{d}{d s} \varphi_{r}(s) d s
\end{aligned}
$$

where $d t / d \varphi_{l}=-t\left(\widetilde{\mathbf{x}}_{i}\right)$ and $d t / d \varphi_{r}=1-t\left(\widetilde{\mathbf{x}}_{i}\right)$.

The integration interval is then transformed to $\xi \in[-1,1]$ to employ a Gauss-Legendre quadrature as described for regular integrals. The two integrals are reduced to only one integral if the projection point $t\left(\widetilde{\mathbf{x}}_{i}\right)$ lies at the element endpoints.

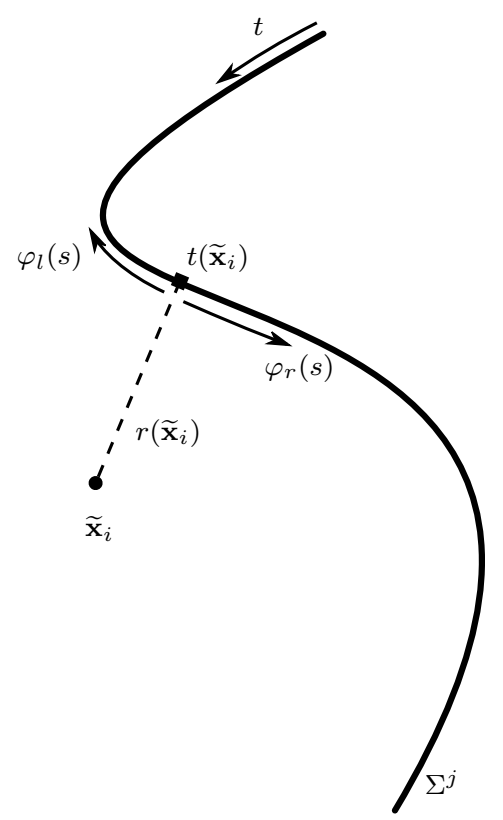

Figure 3: Treatment of singular and weakly singular integral.

We chose a maximum radius $r^{*}$ between the collocation point and the integration element for the identification of singular integrals. Otherwise, the collocation point is sufficiently distant from the integration element and the resulting integrals are regular. The asymptotic behaviour of the fundamental solution is accounted for by selecting the critical radius $r^{*}$ when the integral becomes singular [44]:

$$
\begin{aligned}
& \lim _{r \rightarrow 0} \widetilde{\Psi}=\frac{\iota}{4}+\frac{1}{2 \pi} \log \left(\kappa_{\alpha} r\right) \\
& \lim _{r \rightarrow 0} \frac{\partial \widetilde{\Psi}}{\partial n}=\frac{1}{2 \pi r} \frac{\partial r}{\partial n}
\end{aligned}
$$

Figure 4 shows the fundamental solution for sound pressure and particle normal velocity and its related asymptotic limits. It should be noted that only the real parts present a singularity for the null radius. The critical radius is set from the part where the fundamental solution tends to its asymptotic limit, to give 
$\kappa_{\alpha} r^{*} \approx 0.5$ for the sound pressure solution, whereas for the normal particle velocity it is $\kappa_{\alpha} r^{*} \approx 0.25$. Thus, element integrals become singular when the normal distance from the collocation point to the integration element is $r\left(\widetilde{\mathbf{x}}_{i}\right) \leq r^{*}$. A shaded interval is represented in Figure 4 to indicate where element integrals would have a singular to nearly-singular behaviour. The proposed element integration strategy is also used to compute the domain solution at internal points, which enables the accurate computation of sound pressure close to the boundary.

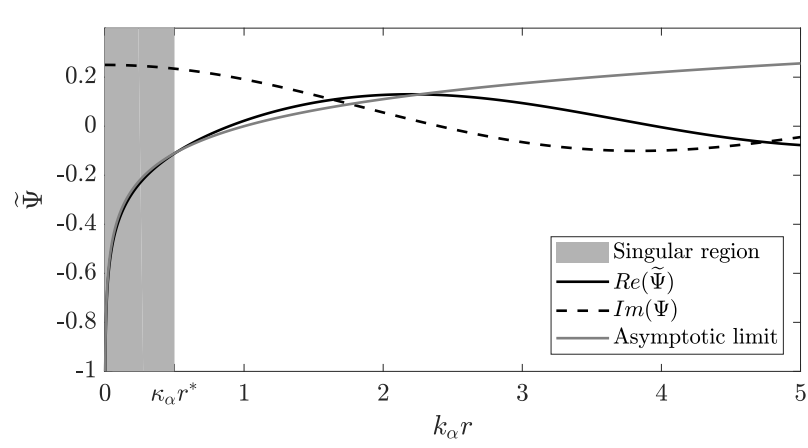

(a)

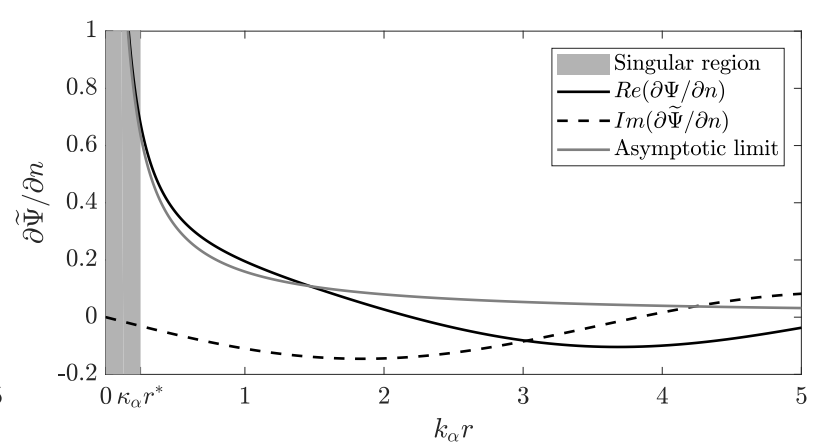

(b)

Figure 4: Representation of the Green's function and intervals for singular integration.

Although the proposed formulation is used to solve the Helmholtz equation, it can be also extended to study more complex problems. The application of the BEM in the Bézier-Bernstein space is quite straightforward for the solution of boundary integral equations involving kernels with strong singularities. However, several problems include hypersingular kernels defined as the Hadamard's finite part instead of a Cauchy principal value.

The proposed method tries to be of global applicability for several problems, so the analytical treatment of hypersingular integrals should discarded since it must necessarily be performed for each kernel and element shape function. Alternatively, researches have shown that regularization processes together with quadrature formulas allow to numerically solve this kind of integral. As an example, Diligenti and Monegato 45] proposed an integration scheme for the implementation of $h p$-BEM using one-dimensional quadrature formulas with an adequate representation of singular and hypersingular kernels. Moreover, Gu et al. [46] presented a comprehensive review of some numerical methods to compute nearly hypersingular integrals in the BEM. To our knowledge, the application of this kind of methods would be straightforward since boundary transformation can be done as explained in this section.

Moreover, the assembled boundary element matrices require special treatment if the boundary has normal discontinuities. Since the sound pressure is unique at any point on the boundary, the element assembling into the matrix $\widetilde{\mathbf{H}}\left(\widetilde{\mathbf{x}}, \kappa_{z}, \omega ; \widetilde{\mathbf{x}}_{i}\right)$ is quite straightforward. However, the particle normal velocity at a discontinuity is not unique. This possibility is taken into account by considering different values according to the element 
normals at this location. However, the compatibility of normal velocities is not achieved when assembling matrix $\widetilde{\mathbf{G}}\left(\widetilde{\mathbf{x}}, \kappa_{z}, \omega ; \widetilde{\mathbf{x}}_{i}\right)$. The assembled matrix has one extra column for each discontinuity point at the boundary and the particle normal velocity vector also has an extra component.

Another possibility commonly used for the treatment of normal discontinuities in potential problems consists of shifting the endpoint nodes inside the element. Thus, the computed solution is discontinuous between elements. Parreira [4] analysed the approximation error for different nodal distribution in discontinuous boundary elements and concluded that the optimal position for nodes inside the elements coincided with Gauss-Legendre integration points. In the next section we show that Chebyshev points of the first kind can be used to define discontinuous elements which simplify the treatment of particle normal velocity at boundary discontinuities. Furthermore, discontinuous elements allow an easy evaluation of the free term beacause the element geometry is smooth at the collocation points, to give $c_{i}\left(\widetilde{\mathbf{x}}_{i}\right)=1 / 2$. Therefore, boundary elements with nodes located at Chebyshev point of the first kind provide a reliable formulation without loss of accuracy as will be seen in next section.

Finally, to conclude this section the proposed $h p$-refinement is described. The solution refinement is quite similar to a standard BEM approach. Boundary elements are subsequently divided using the de Casteljau algorithm and preserving the exact geometry in each refinement without CAD interaction. Moreover, the element order is increased up without comprising the Bézier-Bernstein basis used to describe the boundary geometry. Table 2 summarises the proposed method.

Table 2: Summary of the boundary element method formulated in the Bézier-Bernstein space

A The Bézier-Bernstein space settings:

A.1 Import geometry from CAD model.

A.2 Path subdivision for $h$-refinement through de Casteljau algorithm.

B Field approximation:

B.1 Set element nodes distribution and element order $p$ ( $p$-refinement).

B.2 Compute element shape function from the Lagrangian interpolant relative to the Bernstein basis.

C Boundary element integration:

C.1 Set the critical radius $r^{*}$ for element integration.

C.2 For each collocation point, integration elements further than $r^{*}$ are regularly integrated.

C.3 If any integration element is closer than $r^{*}$, then a smoothing transformation is used for singular integration.

D Solution:

D.1 Boundary solution is obtained by imposing appropriate boundary conditions.

D.2 Domain solution is computed following the same boundary element integration scheme. 


\section{Numerical verification}

In this section, we analyse the performance of the proposed method with a benchmark problem concerning the wave propagation in an unbounded domain in two different configurations: $i$ ) open boundary, and $i i$ ) rigid boundary. An incident pressure field given by $\widetilde{p} I(\widetilde{\mathbf{x}}, \omega)=\exp (\iota \kappa \mathbf{d} \cdot \widetilde{\mathbf{x}})$ was considered in both cases, where the polarised direction was $\mathbf{d}=[1,0]$. This problem has an analytical solution for the two-dimensional Helmholtz equation that is equivalent to setting the longitudinal wave number at $\kappa_{z}=0$. The remaining properties were fluid density $\rho=1.225 \mathrm{~kg} / \mathrm{m}^{3}$ and sound propagation velocity $c_{f}=340 \mathrm{~m} / \mathrm{s}$.

Numerical results were compared with reference solutions and the $L_{2}$ scaled error, $\epsilon_{2}$, was used to assess the accuracy [48:

$$
\epsilon_{2}=\frac{\left\|\widetilde{p}_{e x}\left(\widetilde{\mathbf{x}}, \kappa_{z}, \omega\right)-\widetilde{p}\left(\widetilde{\mathbf{x}}, \kappa_{z}, \omega\right)\right\|}{\left\|\widetilde{p}_{e x}\left(\widetilde{\mathbf{x}}, \kappa_{z}, \omega\right)\right\|}
$$

where $\widetilde{p}_{e x}$ denotes the analytical solution and $\widetilde{p}$ is the numerical result computed by the proposed methodology. Different $h p$-strategies were investigated to get the optimal discretisation with the lowest computational effort. The nodal density per wavelength is used to describe the mesh density [49]:

$$
d_{\lambda}=\frac{2 \pi p}{\kappa h}
$$

where $\kappa$ is the characteristic wavenumber, $h$ is the element size and $p$ is the order of the shape functions. A convergence analysis was carried for several element sizes $h$ with successive $p$-enrichment. Boundary elements were subsequently divided to perform a $h$-refinement and the element order was increased until convergence is reached. For this purpose, we considered that the problem solution was properly approximated if errors satisfied $\log \left(\epsilon_{2}(h, p-1) / \epsilon_{2}(h, p+1)\right) \leq 1$.

\subsection{Case 1: open boundary}

In this example, the Helmholtz equation was solved over a square boundary with sides of $2 \mathrm{~m}$ at a wavenumber $\kappa=7.5 \mathrm{rad} / \mathrm{m}$ (see Figure 5). Four linear Bézier patches were used to define the boundary geometry. The open boundary was set with a Dirichlet condition at the left and right sides and a Neumann condition at the other sides. The problem solution was computed over a grid of receivers inside the boundary allowing a density of twelve points per wavelength. The accuracy of the computed results was assessed using different families of shape functions defined at: $i$ ) equidistant nodes, $i i$ ) LGL integration points, $i i i$ ) Chebyshev points of the first kind, and $i v$ ) Chebyshev points of the second kind. Boundary patches were discretised for different element sizes defined by $\kappa h=7.5, \kappa h=3$ and $\kappa h=1$.

Figure 6 shows the errors in computed results plotted versus the element order and the nodal density per wavelength. The lowest error was $\mathcal{O}\left(10^{-10}\right)$ for interpolation functions defined at Chebyshev points of the second kind for the finest discretisation. However, the solution computed by equidistant nodes had the highest error for a given element size and element order due to deterioration of the numerical results 


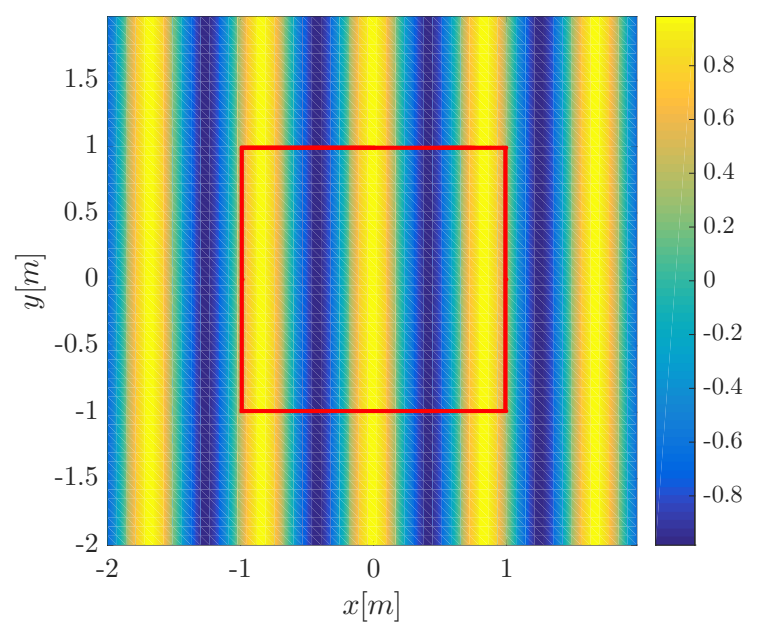

Figure 5: Wave propagation over an unbounded domain. The open boundary is represented in red line.

induced by the Runge phenomenon. The computed results were quite similar for the other interpolant families. This analysis showed that the boundary element formulation based on discontinuous elements defined at Chebyshev points of the first kind is suitable for the easy treatment of normal discontinuities without loss of accuracy.

The convergence was faster for finer discretisation than coarser discretisation. Numerical results converged to the analytical solution when the nodal densities per wavelength were higher than $d_{\lambda}=3$ for the coarsest mesh, $d_{\lambda}=4$ for the medium element size, and $d_{\lambda}=12$ for the smallest element size. Moreover, the solution error for a fixed nodal density was lower for a coarse mesh than a fine mesh, as can be seen in Figure 6.(b). Therefore, a coarse mesh with an adequate nodal density gave better results than a fine mesh with the same density, or equally, the use of high-order elements in coarse meshes ( $p$-refinement) provided better results than low-order elements in a fine discretisation ( $h$-refinement) for a fixed nodal density.

\subsection{Case 2: rigid boundary}

Next, the accuracy in representing boundary geometries in the Bézier-Bernstein space was evaluated by studying the wave scatterering by a fixed cylindrical cavity with radius $r=1 \mathrm{~m}$. The accuracy of the computed results is constrained by the level of precision used to approximate the geometry [50]. In this case, the circular geometry was approximated either by a $C^{1}$ cubic or by a $C^{2}$ quartic Bézier curves 33]. Control points for accurate representation of circular arcs can be found in 33. The performance of the proposed method was compared with the isogeometric approach presented by Peake et al. [51. The analytical solution of this problem can also be found in 33 .

First, the problem solution was computed for a wavenumber $\kappa=40 \mathrm{rad} / \mathrm{m}, \kappa h=3$ and nodal density $d_{\lambda}=10$. The geometry was defined by four cubic patches subdivided into 21 boundary elements of order $p=5$. The element nodes were located at the Chebyshev points of the second kind. The problem solution 


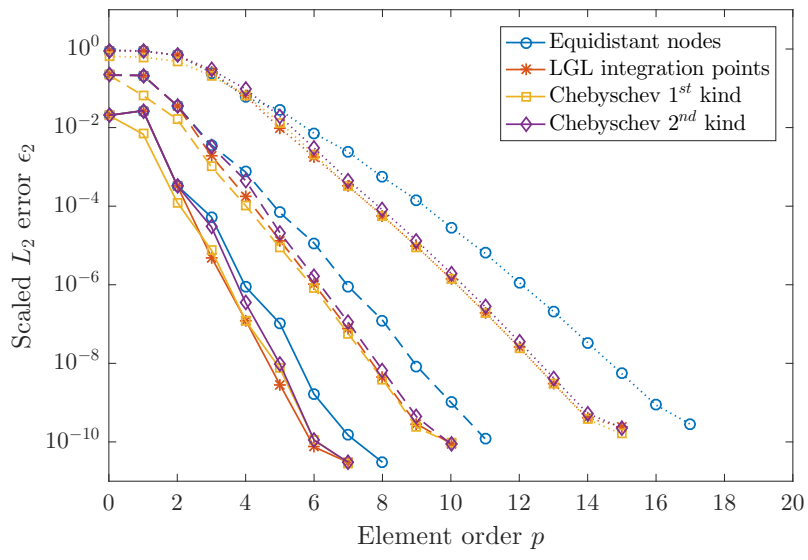

(a)

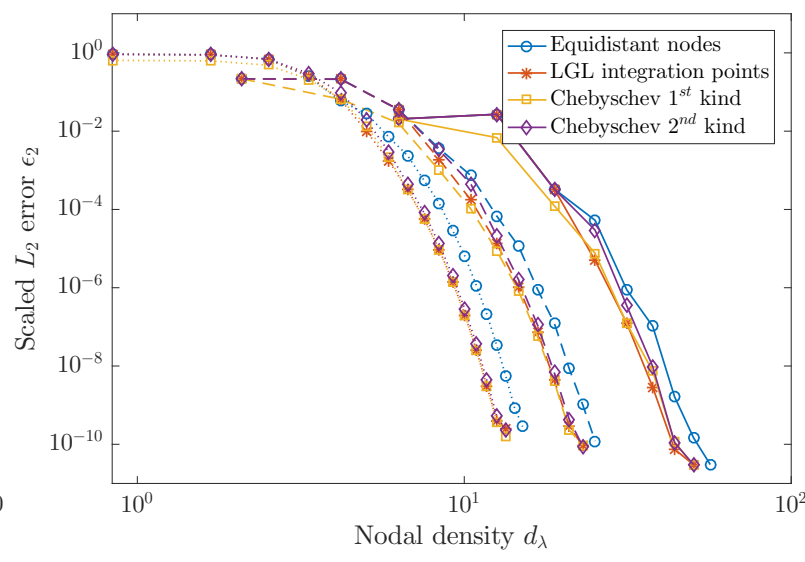

(b)

Figure 6: Convergence of sound pressure using different families of shape functions for $\kappa h=7.5$ (dotted lines), $\kappa h=3$ (dashed lines) and $\kappa h=1$ (solid lines) plotted versus (a) element order $p$ and (b) nodal density per wavelength $d_{\lambda}$.

shows a shadow region behind the cavity, as can be seen in Figure 7.(a). The pressure amplitude over the cavity is represented in Figure 7.(b). Maximum amplitude was reached when the incident wave struck the cavity at point $\theta=\pi \mathrm{rad}$, whereas null pressure was found at the opposite side $(\theta=0 \mathrm{rad})$. The computed results were in very good agreement with the analytical solution [51].

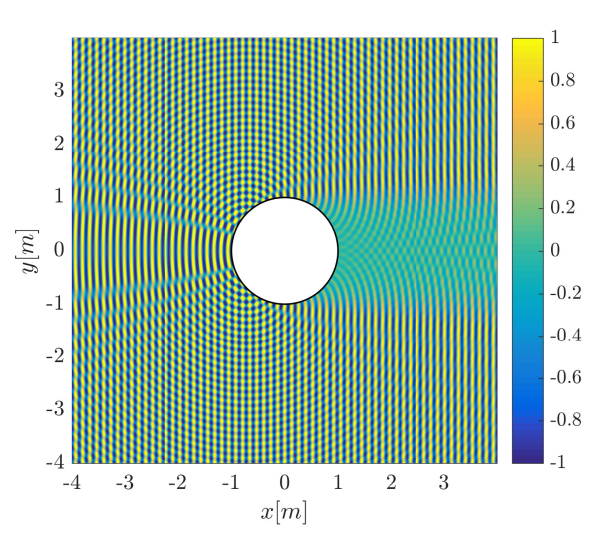

(a)

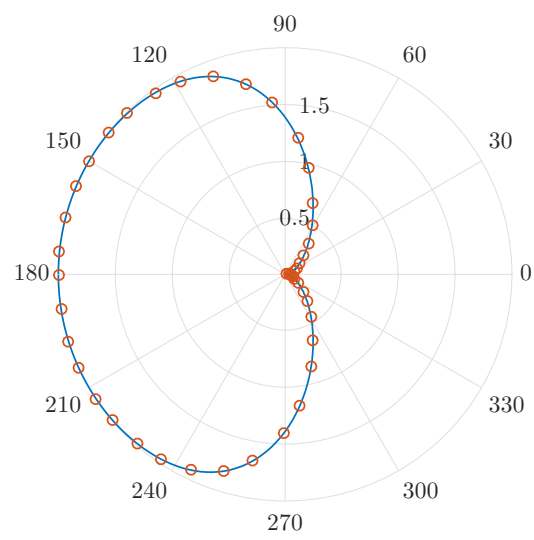

(b)

Figure 7: (a) Real part of wavefield scatterered by a fixed circular cavity at $\kappa=40 \mathrm{rad} / \mathrm{m}$. (b) Pressure amplitude at the boundary.

Thereafter, the solution error over the wavenumber range $\kappa \in(1,400) \mathrm{rad} / \mathrm{m}$ was compared with those obtained by Peake et al. [51] using an isogeometric boundary formulation (IGABEM) and a conventional BEM. The procedure followed by these authors consisted of adding new degrees of freedom to the problem discretisation as the wavenumber increased, to ensure a fixed nodal density $d_{\lambda}=10$. We also followed this 
scheme, starting with three patches at the initial wavenumber $\kappa=1 \mathrm{rad} / \mathrm{m}$. Each patch was subsequently subdivided into elements according to $\kappa h=3$ as the wavenumber increases. The element order was fixed to achieve a nodal density $d_{\lambda}=10$. As an example, Figure 8 represents the related boundary discretisation for $\kappa=1 \mathrm{rad} / \mathrm{m}$ and $\kappa=2.7 \mathrm{rad} / \mathrm{m}$ using $C^{1}$ cubic and $C^{2}$ quartic Bézier curves.

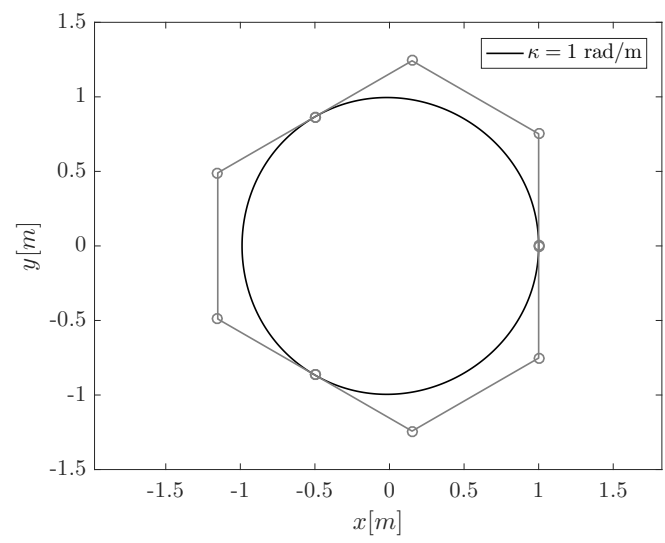

(a)

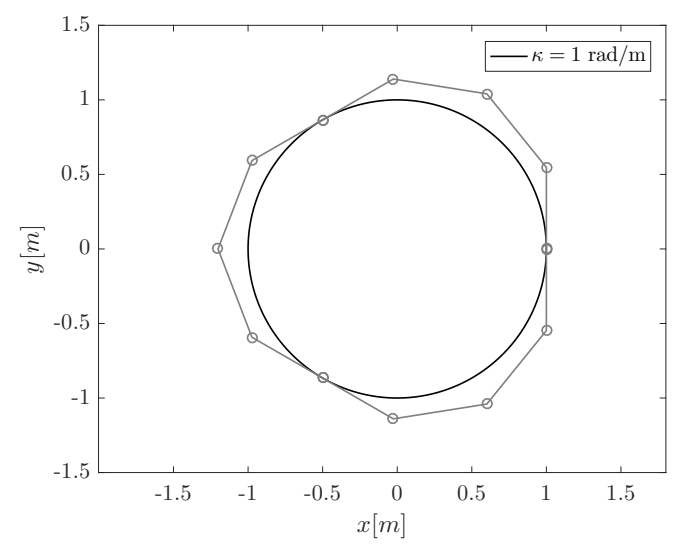

(c)

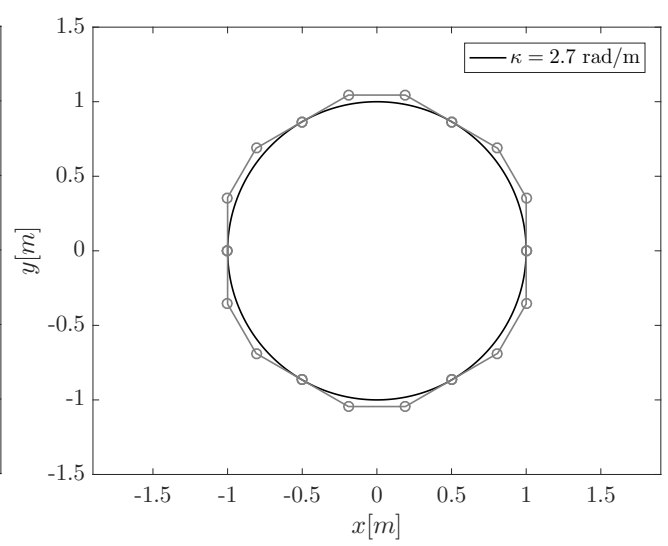

(b)

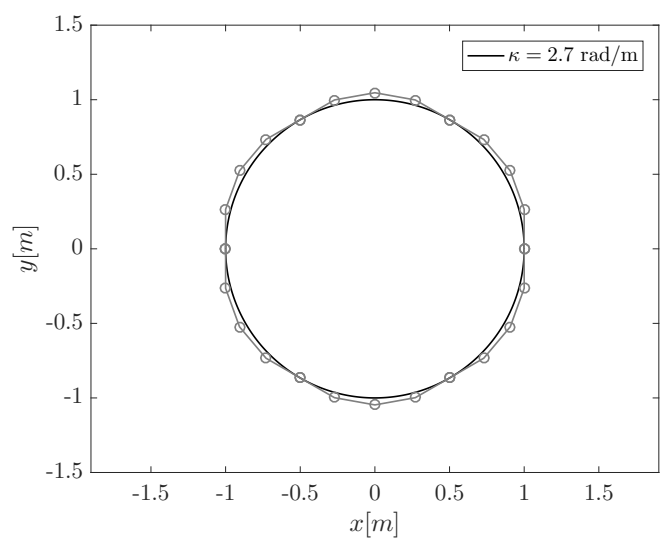

(d)

Figure 8: Circular approximation (black line) with (a,b) $C^{1}$ cubic Bézier curves and (c,d) $C^{2}$ quartic Bézier curves. Control points and their related control polygons are represented by grey circles and grey lines.

Figure 6 shows the scaled $L_{2}$ error $\epsilon_{2}$ of the pressure solution at the boundary. The analysis of the solution error obtained from cubic Bézier curves $(n=3)$ showed a decreasing tendency at low wavenumber until the element size was small enough to accurately represent the circular geometry. Afterwards, the error became uniform of $\mathcal{O}\left(10^{-5}\right)$. The previously mentioned initial effect was avoided when $C^{2}$ quartic Bézier curves $(n=4)$ were used because they can better represent circular arcs. The problem solution deteriorated slightly for higher wavenumbers in both cases, although good results were nonetheless achieved. In general, the proposed method gave more accurate results than the isogeometric formulation and the conventional 
BEM over the studied wavenumber range. However, the solution errors obtained by these methods at high wavenumbers were more uniform than those obtained with the proposed method.

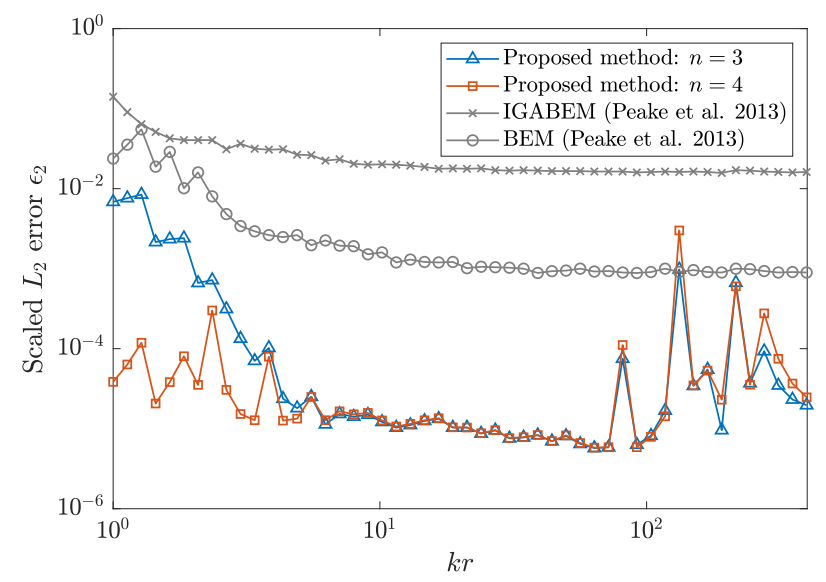

Figure 9: Scaled $L_{2}$ error for different wavenumber compared to IGABEM and BEM [51]

\subsection{A remark on the condition number}

Finally, in this section, we study the condition number for the system of equations in the previous examples. The condition number of BEM matrix is assumed to be moderate for practical applications. It is well known that it increases with the wavenumber $k$ in the solution of the Helmholtz equation due to the pollution effect. Nevertheless, the BEM does not seem to be susceptible to pollution as referred in Reference [52]. Löhndorf and Melenk [52] proved that the $h p$-BEM accuracy is independent of the wavenumber if the condition $k h / p$ is sufficiently small and the element order is $p \geq \mathcal{O}(\log k)$. Although this condition makes it possible to obtain well-posed BEM matrices, in practical applications can produce large systems as the wavenumber increases.

Figures 10 and 11 show the condition number in 2-norm for cases 1 and 2, respectively. The analysis in the first case showed that the condition number remained constant regardless of the element order for a fixed wavenumber. This is true for all families of elements studied except for equidistant nodes. The condition number rapidly increased for element order higher than six due to the Runge phenomenon if the equidistant family of shape function was used.

In the second case, the wavenumber varied in the range $1-400 \mathrm{rad} / \mathrm{m}$. The analysis was carried out with a constant nodal density per wavelength $d_{\lambda}=10$ regardless the wavenumber. This condition leads to a relation $k h / p=0.6$ with a fixed element order $p=5(k h=3)$. Figure 11 shows that the condition wavenumber increased with $k$ and it did not depend on the basis order used for the geometry. This analysis allows us to conclude that the proposed method is at least as well-posed as other formulations to accurately solve this problem, even though the condition given by Löhndorf and Melenk [52] was not achieved due to 
limitations in the system size.

This analysis reveals that the use of independent polynomial basis produces well conditioned systems independently of the approximation developed for geometry and field variable.

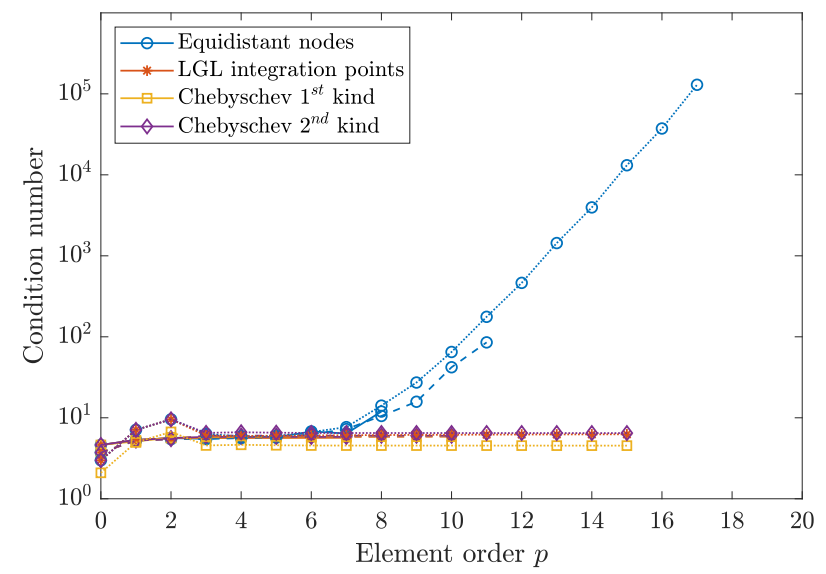

Figure 10: Condition number using different families of shape functions for $\kappa h=7.5$ (dotted lines), $\kappa h=3$ (dashed lines) and $\kappa h=1$ (solid lines) plotted versus element order $p$.

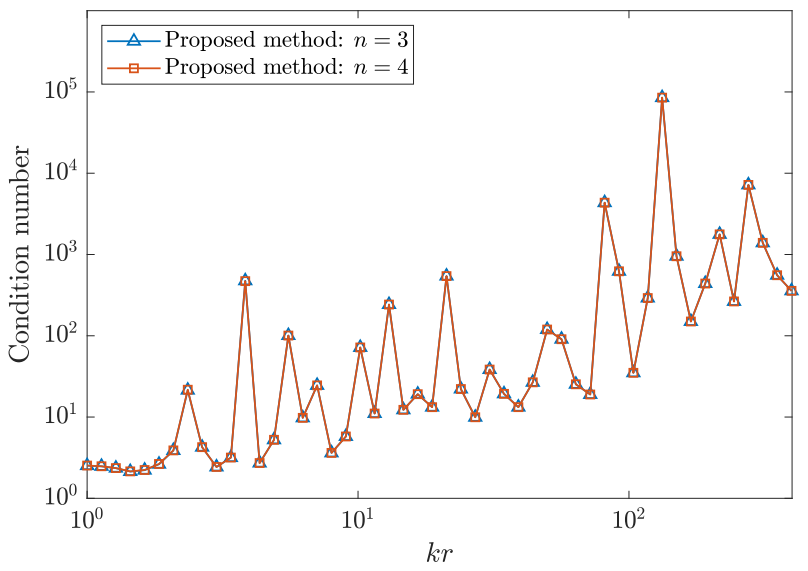

Figure 11: Condition number for different wavenumber.

\section{Numerical example}

In this section, the capability of the proposed method is shown with a numerical example. The problem solved herein studies the three-dimensional wave propagation in a fluid channel with a fixed scatterer. The fluid channel is bounded by two reflection planes that were assumed to be rigid with null particle normal velocity. The total channel height was $H=6 \mathrm{~m}$ and fluid air properties were taken to be those described in the previous section. 
A modified Green's function for the fluid channel was used to limit the discretisation to the scatterer boundary. The method of images was used to construct the modified Green's function [4]. The scatterer and the fluid channel were centred at the origin. The fluid channel and the scatterer were excited by a point source acting at location $\mathbf{x}=(-3,0,0) \mathrm{m}$ with $\kappa=20 \mathrm{rad} / \mathrm{m}$. The source was defined by the modified Green's function. The problem solution was computed in the frequency-wavenumber domain for a longitudinal wavenumber varying over a range from zero to $\kappa_{z}=2 \kappa$ in 128 equidistant steps. The pressure field was evaluated over a grid of receivers with a density of six points per wavelength.

Two geometries were considered: $i$ ) a cylindrical scatterer as shown in Figure 8 and $i i$ ) the complex shaped scatterer represented in Figure 12.(a) directly imported from a CAD model. The geometry of the second example was defined by twelve $C^{1}$ cubic patches. Problem discretisation was given by $\kappa h=6$ and $d_{\lambda}=6$, which leads to an element order $p=6$. The element nodes were located at the Chebyshev points of the second kind. Once the boundary discretisation had been defined, the geometry patches were subdivided as shown in Figure 12(b). The cylindrical scatterer was discretised following a similar procedure.

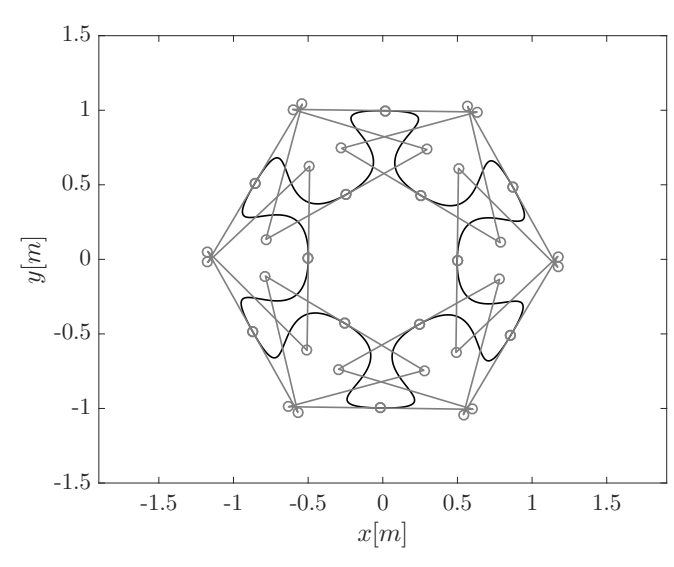

(a)

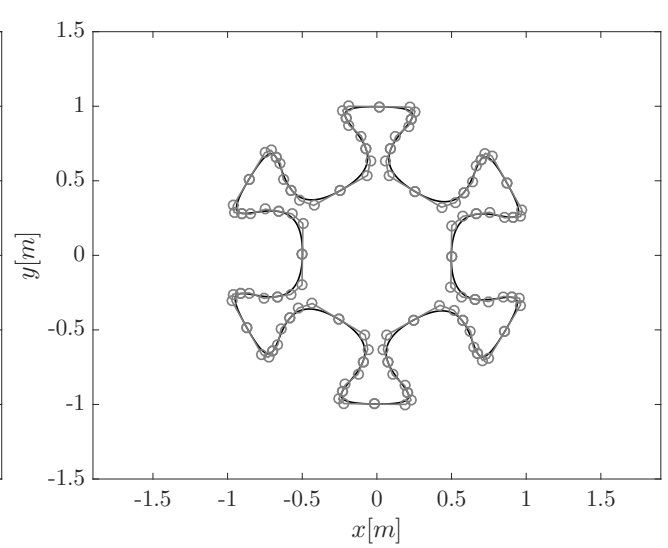

(b)

Figure 12: (a) Complex geometry imported from CAD model and (b) related discretisation for $\kappa h=6$. Control points and their related control polygons are represented by grey circles and grey lines.

Figure 13 shows the incident wavefield and the scatterered wave. The representation is limited to a half model, according to the problem symmetry. The incident pressure field shows a spherical distribution that was gradually lost as the distance from the source point increased due to channel wave refraction (Figure 13.(a)). The amplitude decreased with the distance to the excitation point, according to the radiation condition. The pattern of the incident wave changed under the effect of the scatterers. A complex pattern was found, caused by waves reflected from both the channel borders and the scatterer boundary. A shadow region was found behind the scatterers where pressure amplitude was considerably lower.

This example has shown that the proposed method is, therefore, a useful tool for studying boundary 
problems with exact CAD geometry. An independent geometry and variable field approximation allowed efficient modelling that took both the complexity of the boundary shape and the studied wavenumber into account. In this case, it was enough to use cubic curves for the geometry approximation, while it was necessary to use sixth order elements for the variable field. 


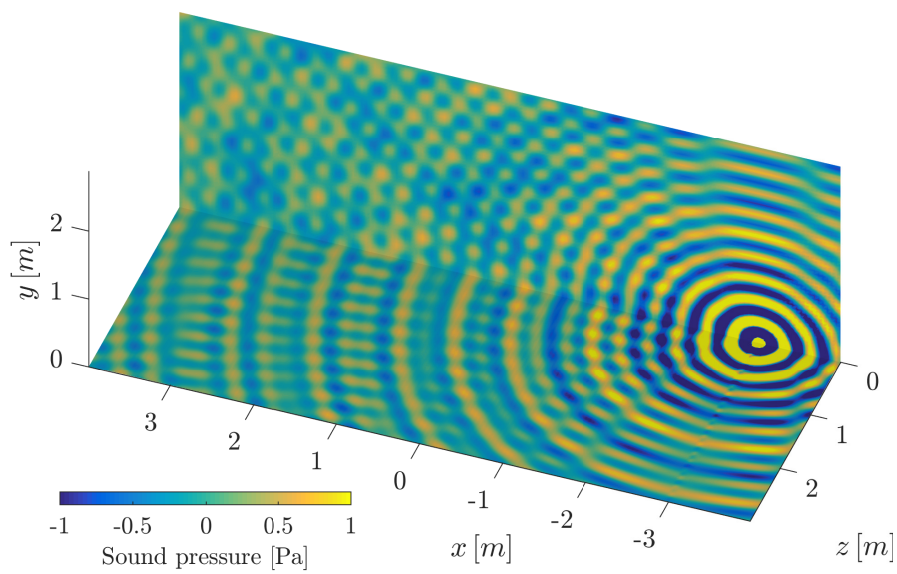

(a)

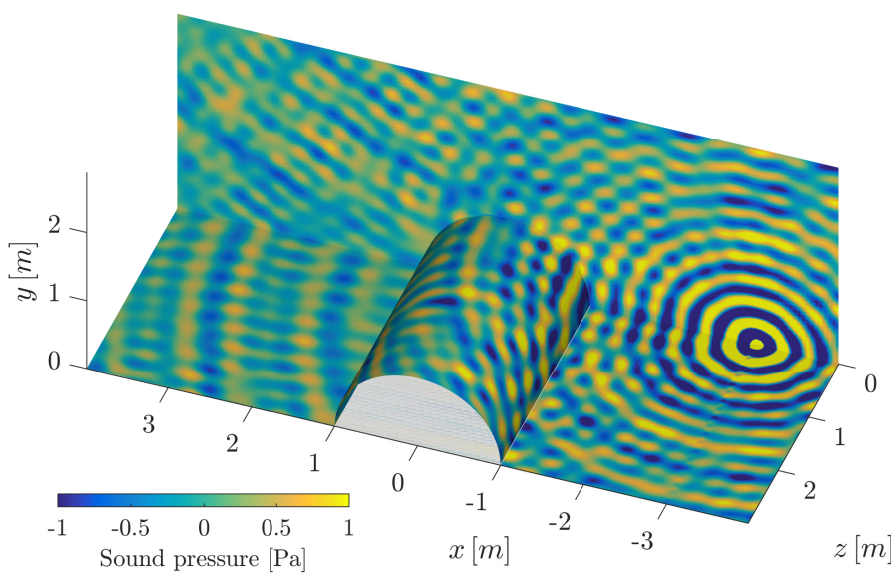

(b)

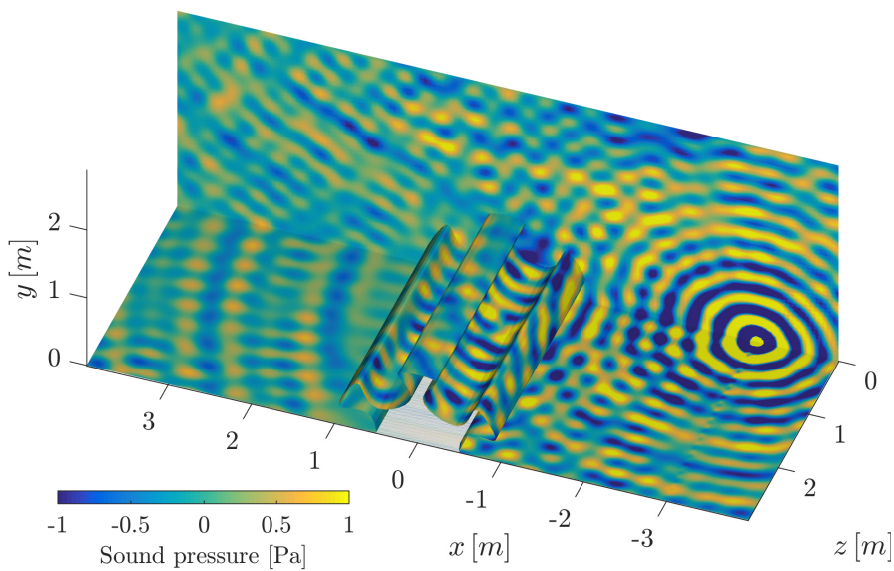

$(25)$

Figure 13: Real part of (a) incident wavefield, and the scatterered wavefield by (b) a cylindrical cavity, and (c) a complex shape boundary. 


\section{Conclusions}

This paper has proposed a novel formulation of the BEM to consider exact CAD geometry. The boundary geometry is described in the Bézier-Bernstein space which is commonly used in computer-aided design. Boundary elements are defined by the subdivision of Bézier curves according to an $h$-refinement strategy. The element geometry is evaluated and subdivided using the de Casteljau algorithm and the polar form of Bernstein polynomials. Geometric operations are easily performed without CAD interaction which entails a great flexibility for $h$-refinement.

The field variables are approximated independently of the geometry representation. Therefore, the proposed method is arbitrarily defined as subparametric, isoparametric or superparametric, depending on the problem properties and boundary geometry. The approximation of field variables has been generalised considering different shape functions families defined from node locations and element degree, using the Newton-Bernstein algorithm.

The boundary element integrals are solved according to distance from collocation points and the integration element. The asymptotic behaviour of the fundamental solution has given a critical radius below which the element integrals become singular. In this case, singular integrals have been numerically integrated with a polynomial transformation of the integration domain.

The proposed method has been verified using two benchmark problems with known analytical solution. Different families of shape functions have been tried and numerical results obtained have accurately approximated the problem solution. The Lagrangian interpolant relative to the Bernstein basis defined at Chebyshev points of the first kind was suitable for implementing discontinuous elements to treat sharp corners. Finally, the capability of the proposed method has been shown in the solution of wave scatterering through a fluid channel in presence of a fixed cavity of irregular shape.

In particular, this work has been used to solve the Helmholtz equation in longitudinally invariant domains, but it is also possible to apply it in different two-dimensional or 2.5D physical problems, using an appropriate fundamental solution for the boundary element method.

The proposed method keeps the simplicity of the standard BEM and is able to solve more challenging problems with complex geometry shape. Future developments on this topic include its extension to both purely 3D geometry (see Reference [23]) and multi-physics problems (e.g. elasticity, heat transfer, coupled problems).

\section{Acknowledgements}

The research work presented herein was supported by the Spanish Ministry of the Economy and Competitiveness (Ministerio de Economía y Competitividad) through research project BIA2016-75042-C2-1-R. It was also supported by project POCI-01-0247-FEDER-017759, funded by Portugal 2020 through the Operational 
Programme for Competitiveness Factors (COMPETE 2020). Financial support is gratefully acknowledged. The authors also wish to acknowledge the support provided by the Andalusian Scientific Computing Centre (CICA).

\section{References}

[1] E. Atroshchenko, S.P.A. Bordas, Geometry-independent field approximation for spline-based finite element methods, 11th World Congress on Computational Mechanics, 2014.

[2] J. Domínguez, Boundary elements in dynamics, Computational Mechanics Publications and Elsevier Aplied Science, Southampton, 1993.

[3] M. Bonnet, G. Maier, C. Polizzotto, Symmetric Galerkin Boundary Element Methods, Applied Mechanics Reviews 51 (11) (1998) 669-704.

[4] Y. Liu, S. Mukherjee, N. Nishimura, M. Schanz, W. Ye, A. Sutradhar, E. Pan, N. Dumont, A. Frangi, A. Saez, Recent advances and emerging applications of the Boundary Element Method, Applied Mechanics Reviews 64 (3).

[5] D. Poljak, D. Cavka, H. Dodig, C. Peratta, A. Peratta, On the use of the boundary element analysis in bioelectromagnetics, Engineering Analysis with Boundary Elements 49 (2014) 2-14.

[6] Y. Gu, X. He, W. Chen, C. Zhang, Analysis of three-dimensional anisotropic heat conduction problems on thin domains using an advanced boundary element method, Computers and Mathematics with Applications 75 (1) (2018) 33-44.

[7] A. Zhang, Y. Gu, Q. Hua, W. Chen, C. Zhang, A regularized singular boundary method for inverse Cauchy problem in three-dimensional elastostatics, Advances in Applied Mathematics and Mechanics 10 (6) (2018) 1459-1477.

[8] Y. Gu, C.-M. Fan, R.-P. Xu, Localized method of fundamental solutions for large-scale modeling of two-dimensional elasticity problems, Applied Mathematics Letters 93 (2019) 8-14.

[9] L. De Lorenzis, P. Wriggers, T.J.R. Hughes, Isogeometric contact: A review, GAMM Mitteilungen 37 (1) (2014) 85-123.

[10] D. Schillinger, M. Ruess, The Finite Cell Method: A Review in the Context of Higher-Order Structural Analysis of CAD and Image-Based Geometric Models, Archives of Computational Methods in Engineering 22 (3) (2015) $391-455$.

[11] B. Marussig, T.J.R. Hughes, A Review of Trimming in Isogeometric Analysis: Challenges, Data Exchange and Simulation Aspects, Archives of Computational Methods in Engineering (2017) 1-69.

[12] B. Urick, T.M. Sanders, S.S. Hossain, Y. Zhang, T.J.R. Hughes, Review of Patient-Specific Vascular Modeling: TemplateBased Isogeometric Framework and the Case for CAD, Archives of Computational Methods in Engineering (2017) 1-24.

[13] M. Sehmi, J. Christensen, C. Bastien, S. Kanarachos, Review of topology optimisation refinement processes for sheet metal manufacturing in the automotive industry, Structural and Multidisciplinary Optimization 58 (1) (2018) 305-330.

[14] T.J.R. Hughes, J.A. Cottrell, Y. Bazilevs, Isogeometric analysis: CAD, finite elements, NURBS, exact geometry and mesh refinement, Computer Methods in Applied Mechanics and Engineering 194 (39-41) (2005) 4135-4195.

[15] D. Wang, J. Xuan, An improved NURBS-based isogeometric analysis with enhanced treatment of essential boundary conditions, Computer Methods in Applied Mechanics and Engineering 199 (37-40) (2010) 2425-2436.

[16] M.A. Scott, R.N. Simpson, J.A. Evans, S. Lipton, S.P.A. Bordas, T.J.R. Hughes, T.W. Sederberg, Isogeometric boundary element analysis using unstructured T-splines, Computer Methods in Applied Mechanics and Engineering 254 (2013) $197-221$.

[17] H. Casquero, L. Liu, Y. Zhang, A. Reali, H. Gomez, Isogeometric collocation using analysis-suitable T-splines of arbitrary degree, Computer Methods in Applied Mechanics and Engineering 301 (2016) 164-186.

[18] H. Lian, P. Kerfriden, S.P.A. Bordas, Implementation of regularized isogeometric boundary element methods for gradientbased shape optimization in two-dimensional linear elasticity, International Journal for Numerical Methods in Engineering 106 (12) (2016) 972-1017. 
[19] X. Peng, E. Atroshchenko, P. Kerfriden, S.P.A. Bordas, Isogeometric boundary element methods for three dimensional static fracture and fatigue crack growth, Computer Methods in Applied Mechanics and Engineering 316 (2017) 151-185.

[20] R.W. Johnson, Higher order b-spline collocation at the greville abscissae, Applied Numerical Mathematics 52 (1) (2005) 63-75.

[21] E. Zieniuk, A new integral identity for potential polygonal domain problems described by parametric linear functions, Engineering Analysis with Boundary Elements 26 (10) (2002) 897-904.

[22] E. Zieniuk, A. Boltuc, Bézier curves in the modeling of boundary geometry for 2D boundary problems defined by Helmholtz equation, Journal of Computational Acoustics 14 (3) (2006) 353-367.

[23] E. Zieniuk, K. Szerszeń, Nonelement boundary representation with Bézier surface patches for 3D linear elasticity problems in parametric integral equation system (PIES) and its solving using Lagrange polynomials, Numerical Methods for Partial Differential Equations 34 (1) (2018) 51-79.

[24] J. Hoschek, D. Lasser, Fundamentals of Computer Aided Geometric Design, A. K. Peters, Ltd., Natick, MA, USA, 1993.

[25] R.T. Farouki, The Bernstein polynomial basis: A centennial retrospective, Computer Aided Geometric Design 29 (6) (2012) 379-419.

[26] R.T. Farouki, V.T. Rajan, Algorithms for polynomials in Bernstein form, Computer Aided Geometric Design 5 (1) (1988) $1-26$.

[27] R.T. Farouki, Legendre-Bernstein basis transformations, Journal of Computational and Applied Mathematics 119 (1-2) (2000) 145-160.

[28] M. Bouchon, K. Aki, Discrete wave-number representation of seismic source wavefields, Bulletin of the Seismological Society of America 67 (2) (1977) 259-277.

[29] A. Romero, A. Tadeu, P. Galvín, J. António, 2.5D coupled BEM-FEM used to model fluid and solid scatterering wave, International Journal for Numerical Methods in Engineering 101 (2) (2015) 148-164.

[30] L. Ramshaw, Blossoming: A connect-the-dots approach to splines, Digital Equipment Corporation SRC Report No. 19.

[31] H.-P. Seidel, An Introduction to Polar Forms, IEEE Comput. Graph. Appl. 13 (1) (1993) 38-46.

[32] T. Dokken, M. Dæhlen, T. Lyche, K. Mørken, Good approximation of circles by curvature-continuous Bézier curves, Computer Aided Geometric Design 7 (1-4) (1990) 33-41.

[33] S. Hur, T.-W. Kim, The best G1 cubic and G2 quartic Bézier approximations of circular arcs, Journal of Computational and Applied Mathematics 236 (6) (2011) 1183-1192.

[34] J.-P. Berrut, L.N. Trefethen, Barycentric Lagrange interpolation, SIAM Review 46 (3) (2004) 501-517.

[35] M. Ainsworth, M.A. Sánchez, Computing the Bézier control points of the lagrangian interpolant in arbitrary dimension, SIAM Journal on Scientific Computing 38 (3) (2016) A1682-A1700.

[36] A.T. Patera, A spectral element method for fluid dynamics: Laminar flow in a channel expansion, Journal of Computational Physics 54 (3) (1984) 468-488.

[37] B. Fornberg, A Practical Guide to Pseudospectral Methods, Cambridge Monographs on Applied and Computational Mathematics, Cambridge University Press, 1996.

[38] R.T. Farouki, T.N.T. Goodman, T. Sauer, Construction of orthogonal bases for polynomials in Bernstein form on triangular and simplex domains, Computer Aided Geometric Design 20 (4) (2003) 209-230.

[39] A. Marco, J.-J. Martínez, A fast and accurate algorithm for solving Bernstein-Vandermonde linear systems, Linear Algebra and Its Applications 422 (2-3) (2007) 616-628.

[40] A. Marco, J.-J. Martínez, Accurate computations with totally positive Bernstein-Vandermonde matrices, Electronic Journal of Linear Algebra 26 (2013) 357-380.

[41] A. Marco, J.-J. Martínez, R. Viana, Accurate polynomial interpolation by using the Bernstein basis, Numerical Algorithms 75 (3) (2017) 655-674. 
[42] A. Romero, P. Galvín, J. António, J. Domínguez, A. Tadeu, Modelling of acoustic and elastic wave propagation from underground structures using a 2.5D BEM-FEM approach, Engineering Analysis with Boundary Elements 76 (2017) 26-39.

[43] G. Monegato, L. Scuderi, Numerical integration of functions with boundary singularities, Journal of Computational and Applied Mathematics 112 (1-2) (1999) 201-214.

[44] T. Wu, Boundary Element Acoustics Fundamentals and Computer Codes, Advances in Boundary Elements, WIT Press, 2000.

[45] M. Diligenti, G. Monegato, Integral evaluation in the BEM solution of (hyper)singular integral equations. 2D problems on polygonal domains, Journal of Computational and Applied Mathematics 81 (1) (1997) $29-57$.

[46] Y. Gu, Q. Hua, W. Chen, C. Zhang, Numerical evaluation of nearly hyper-singular integrals in the boundary element analysis, Computers and Structures 167 (2016) 15-23.

[47] P. Parreira, On the accuracy of continuous and discontinuous boundary elements, Engineering Analysis 5 (4) (1988) 205-211.

[48] S. Petersen, D. Dreyer, O. von Estorff, Assessment of finite and spectral element shape functions for efficient iterative simulations of interior acoustics, Computer Methods in Applied Mechanics and Engineering 195 (44-47) (2006) 6463 6478.

[49] H. Bériot, G. Gabard, E. Perrey-Debain, Analysis of high-order finite elements for convected wave propagation, International Journal for Numerical Methods in Engineering 96 (11) (2013) 665-688.

[50] D.S. Meek, Coaxing a planar curve to comply, Journal of Computational and Applied Mathematics 140 (1-2) (2002) 599-618.

[51] M.J. Peake, J. Trevelyan, G. Coates, Extended isogeometric boundary element method (XIBEM) for two-dimensional Helmholtz problems, Computer Methods in Applied Mechanics and Engineering 259 (2013) 93-102.

[52] M. Löhndorf, J. Melenk, Wavenumber-explicit $h p$-BEM for high frequency scattering, SIAM Journal on Numerical Analysis 49 (6) (2011) 2340-2363. 